\title{
PIV MEASUREMENTS OF TURBULENT FLOWS IN A 61-PIN WIRE-WRAPPED HEXAGONAL FUEL BUNDLE
}

Thien Nguyen, Nolan Goth, Philip Jones, Saya Lee, Rodolfo Vaghetto, Yassin Hassan

Department of Nuclear Engineering, Texas A\& M University

3133 TAMU, College Station, TX 77801, USA

\begin{abstract}
The current work experimentally investigates the flow characteristics in the near-wall region of the 61-pin wire-wrapped hexagon fuel bundle via the matched-index-of-refraction technique. Particle image velocimetry (PIV) measurements were taken in the region near the surfaces of the pins, wires and enclosure wall at the Reynolds number of 19,000. From the obtained PIV velocity vector fields, flow statistics such as mean velocity and rootmean-square fluctuating velocity profiles were computed. In addition, spatial-temporal crosscorrelations of velocity-velocity and pressure-velocity were analyzed. A strong correlation between the wall fluctuating pressure signal and flow structures was observed. Finally, we applied the POD analysis to the vorticity snapshots obtained in the near-wall region to reveal the dominant flow structures. It was found that the large-scale structures were elongated and aligned with the mean flow direction.
\end{abstract}

\section{Nomenclature}

〈. time-averaged operator

$\boldsymbol{\omega} \quad$ vorticity $\left(\mathrm{s}^{-1}\right)$

$\eta \quad$ separation length $(\mathrm{mm})$

$\mu_{p c y} \quad$ dynamic viscosity of p-cymene $(\mathrm{Pa} \times \mathrm{s})$

$\nu_{p c y} \quad$ kinematic viscosity of p-cymene $\left(\mathrm{m}^{2} / \mathrm{s}\right)$

$\rho_{p c y} \quad$ density of p-cymene $\left(\mathrm{kg} / \mathrm{m}^{3}\right)$

Email address: thien.duy.ng@tamu.edu (Thien Nguyen) 
$\tau \quad$ time delay (s)

$C_{x y}(f)$ squared coherent function between points $x$ and $y$

$P_{x y}(f)$ power spectral density between points $x$ and $y$

$R_{p v} \quad$ pressure-velocity spatial-temporal cross-correlation coefficients

$R_{u u 0}, R_{v v 0}$ velocity-velocity spatial cross-correlation coefficients

$R_{u u}, R_{v v}$ velocity-velocity spatial-temporal cross-correlation coefficients

Re $=U_{m} \times D_{h} / \nu$, Reynolds number

$\Delta t \quad$ PIV inter-frame time delay (s)

$A_{f} \quad$ cross-section flow area $\left(\mathrm{cm}^{2}\right)$

$D_{e} \quad$ eddy size $(\mathrm{mm})$

$D_{h} \quad$ fuel bundle hydraulic diameter $(\mathrm{mm})$

f $\quad$ sampling frequency $(\mathrm{Hz})$

$L \quad$ inner duct size length of the hexagonal enclosure $(\mathrm{mm})$

$L_{x}, L_{y}$ integral length scales $(\mathrm{mm})$

$N \quad$ number of samples

$p \quad$ wall fluctuating pressure $(\mathrm{Pa})$

$P_{d} \quad$ fuel pin diameter $(\mathrm{mm})$

$P_{t} \quad$ pitch length of fuel pin $(\mathrm{mm})$

$P_{w} \quad$ total wetted perimeter $(\mathrm{m})$

$U, V$ horizontal and vertical time-averaged velocities $(\mathrm{m} / \mathrm{s})$

$u^{\prime}, v^{\prime}$ horizontal and vertical fluctuating velocities $(\mathrm{m} / \mathrm{s})$

$U_{c} \quad$ convection velocity $(\mathrm{m} / \mathrm{s})$

$U_{m} \quad$ mean bulk flow velocity $(\mathrm{m} / \mathrm{s})$

$W_{d} \quad$ helical wire diameter $(\mathrm{mm})$

$x, y$ horizontal (traversal), vertical (axial) directions 


\section{Introduction}

Liquid metal fast reactors using sodium as a coolant typically utilize a tightly packed triangular lattice of fuel pins enclosed in a hexagonal duct. The fuel pin is typically wrapped with a helical wire spacer. The primary function of the wire spacers is to maintain a gap between neighboring fuel pins. They also mitigate vortex-induced vibration and enhance subchannel mixing to increase convective heat transfer (Fischer et al, 2007). It is, however, noted in the work of Saxena et al. (2013) that hot spots and a cyclic temperature variation may appear and adversely affect the clad properties because of thermal strippings. On the other hands, results from Pointer et al. (2008) have shown that the helical wires in the bundles induced the traversal flow and enhanced the heat transfer. In addition, these authors noted that the presence of wires created a wake behind, increased the pressure drop and turbulence level. It is noticed that the contributions of helical wires in the fuel bundle are arguable, numerical and experimental investigations of the flow in the fuel assembly are essential.

Many experiments have been performed to study thermal-hydraulics characteristics, primarily pressure drops, of the wire-wrapped fuel bundles. Novendstern (1972) developed a semi-empirical model to predict pressure losses in a hexagonal array of wire-wrapped pins in the turbulent flow regime. Rehme (11973) proposed pressure drop correlations for a wide range of geometrical parameters, such as pitch-to-diameter ratio $P / D$ from 1.125 to 1.417 , lead-to-wire diameter ratio from 6 to 45, and number of pins $n$ from 7 to 61. Cheng and Todreas (1986) introduced their hydrodynamic models for sub-channel friction factors and mixing parameters in a 37-pin fuel bundle, that covered the laminar, transition and turbulent flow regimes. Computational fluid dynamics (CFD) offers an opportunity to validate the above correlations and investigate the flow characteristics in the sub-channels. Several numerical studies of the wire-wrapped fuel bundles were performed, and most of them were Reynolds-Averaged Navier-Stokes (RANS) based simulations with various turbulence models. Ahmad and Kim (2006) performed RANS simulations of 7- and 19-pin bundles using the k- $\omega$ SST turbulent model, while Rolfo et al. (2012) studied the heat transfers in the 7-, 19- and 61-pin bundles using their RANS simulations. Gajapathy et al. (2015) performed RANS simulations on 7-pin bundles using the k- $\epsilon$ model. A benchmark of RANS calculations for the 7-pin fuel bundle among several groups was discussed in Merzari et al. (2016). Recently, RANS calculations using $\mathrm{k}-\epsilon$ and $\mathrm{k}-\omega$ turbulence models for 37-pin fuel bundle were presented in Rasu et al. (2014) and Jeong et al. (2015). For transient calculations, Fischer et al. (2007) carried out large-eddy simulation (LES) calculations on the wire-wrapped fuel bundle to obtain higher-resolution numerical of sub-channel behaviors.

The geometrical complexity of the hexagonal wire-wrapped fuel bundle has limited exper- 
imental activities to pressure drop measurements. A database of pressure drops and flow-field measurements in a 61-pin wire-wrapped hexagonal fuel bundle is not available to benchmark the existing correlations and validate the CFD calculations. Researchers at Texas A\&M University have conducted isothermal flow experiments in a wire-wrapped 61-pin hexagonal fuel bundle to support the research on the advanced nuclear fuel development sponsored by the U.S. Department of Energy (DOE). The general purpose of these tests is to perform high spatial and temporal resolution measurements of the pressure and velocity fields at different locations in the fuel bundle. The experimental activities provide an experimental database of the pressure and flowfield measurements suitable for validating CFD computer codes.

The objective of this paper is to investigate the flow characteristics in the near-wall region of the 61-pin wire-wrapped hexagon fuel bundle at a Reynolds number $R e=19,000$. The wire-wrapped experimental facility employed the matched-index-of-refraction (MIR) technique to allow laser diagnostic velocity measurement techniques, for example, particle image velocimetry (PIV) discussed in this paper. The 61-pin wire-wrapped fuel bundle facility and PIV experimental setup are presented in Sect. 2 , and followed by the experimental results in Sect. 3. The statistics results obtained from the PIV measurements are presented in Sect. [3.], while the analysis of two-point cross-correlation is discussed in Sect. 3.2. To reveal the most dominant flow structures in the near-wall region that play an important role in the flow dynamics and heat transfer, proper orthogonal decomposition (POD) is performed on the PIV vorticity fields. Results from the vorticity decomposition are discussed in Sect. 3.3.

\section{Experimental facility and PIV experimental setup}

\subsection{Experimental facility of 61-pin bundle}

In this section, we describe the experimental facility of 61-pin hexagonal fuel bundle with helically wrapped wire spacers. Figure $\mathbb{0}(\mathrm{a})$ shows an overview the experimental facility which consisted of a primary loop for providing the main fluid flow for the experiments, and a secondary loop for performing various controls of volume rate, temperature, and filtration. Primary components and their uses are described in the followings.

- A primary tank (1) to store the working fluid and as an inline surge volume.

- A primary pump (2) and an associated variable frequency drive (VFD) to supply and control the flow rate in the test section, respectively.

- An inline turbine flow meter (3) and a resistance temperature detector (4) (RTD). 

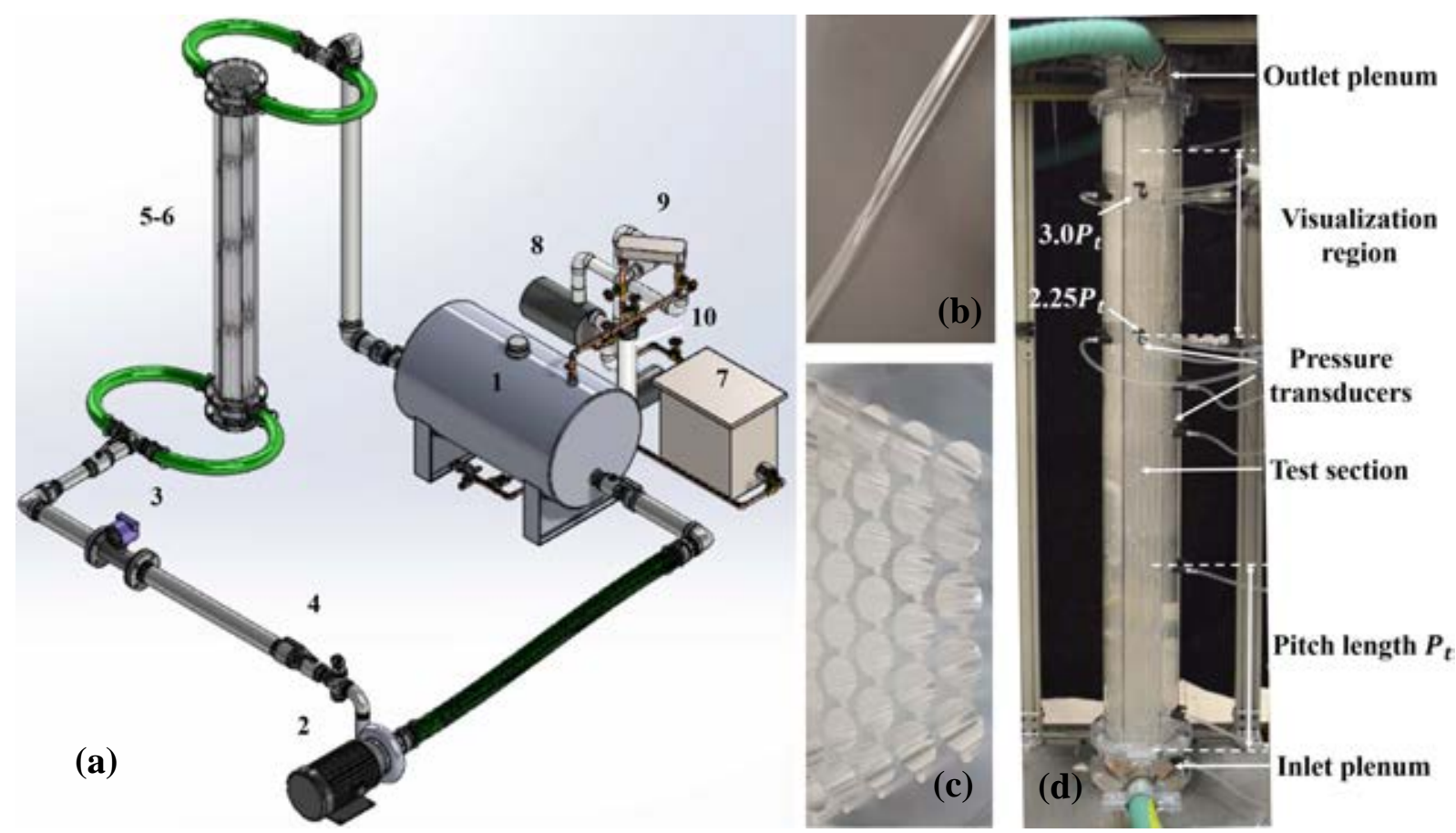

Figure 1: An overview of the experimental facility of 61-pin hexagonal fuel bundle with helically wrapped wire spacers. (a) Experimental flow loop design, (b) photo of a wirewrapped pin, (c) bottom plate designed to support the pins, and (d) test section.

- The hexagonal test section containing the 61-pin bundle (5) with nine pressure transducers mounted and additional differential pressure transducers (6) (pressure transducers are not visible in the figure).

The secondary loop included a secondary tank (7), a secondary pump (8) with associated VFD, a heat exchanger (9) supplied with a water chiller to maintain the temperature in the primary loop, and a filtration system (10). Since the experimental facility was designed to operate with water and p-cymene, all components were made of chemical-resistant materials. Several attempts were made in order to obtain the best design and connection techniques applied to junctions of the test section, and the loop to minimize mechanical vibrations. In the current design of the experimental facility, fluid flow in the test section was driven by the primary centrifugal pump, whose flowrates ranged from 50 to 300 gallons per minute (GPM).

The test section shown in figure $\mathrm{W}(\mathrm{d})$ had the total axial height of 1,666 (mm) excluding the inlet and outlet plena located at the bottom and the top of the bundle, respectively. The hexagonal enclosure consisted of 61 helically wrapped pins (c.f. figure $\mathrm{m}(\mathrm{b})$ ) that were arranged in a triangular lattice. The inner duct side length of the hexagonal enclosure was 
$L=88.8(\mathrm{~mm})$. The pin and wire diameters were $P_{d}=15.9(\mathrm{~mm})$ and $W_{d}=3(\mathrm{~mm})$, respectively, yielding the dimensionless ratio $\left(P_{d}+W_{d}\right) / P_{d}=1.189$. The pitch length of the wire and pin was $P_{t}=476(\mathrm{~mm})$. The calculated flow area was $A_{f}=79.86\left(\mathrm{~cm}^{2}\right)$ while the total wetted perimeter was $P_{w}=4.15(\mathrm{~m})$ yielding the hydraulic diameter $D_{h}=7.7(\mathrm{~mm})$. The pins were attached to a bottom plate (see figure $\mathbf{\square}(\mathrm{c})$ ), that was connected to the inlet plenum, and to a top plate included in the outlet plenum. The flow entered the inlet plenum, and exited the outlet plenum symmetrically from the two sides of each plenum via 2-inch diameter flexible pipes. It is important to note that the current configuration of the 61-pin fuel bundle was designed based on the preliminary CFD calculations, and the experimental results were used to support validations and provide benchmarks for numerical calculations. With the supported numerical results, the test section was built with 2.5 wire pitch axial length to allow for fully-developed flow without the need of using honeycomb grid and straight duct, and to minimize any reverse flow from the outlet. The flow measurements were performed in the visualization region which started from $2.25 P_{t}$ to $3.25 P_{t}$ (one full wire pitch length). The pins, wires, and hexagonal duct enclosure were made of transparent poly-methyl methacrylate to allow for flow visualization and measurements using laser-based techniques. Along the axial length, nine pressure transducers and several differential pressure transducers were installed on the hexagonal exterior surfaces via 1/4 inch NPT threaded holes to measure the pressure drops within the fuel bundle. Results from the pressure drop measurements will be presented in another paper. The current work will discuss the correlation between fluctuating pressure signals measured by two dynamic pressure transducers and velocity fields obtained by the PIV measurements. Details of the experimental setups for the pressure and velocity measurements are described in the next sections.

\subsection{PIV experimental setup}

Figure $\square$ shows an experimental configuration of the two-dimensional two-component (2D2C) PIV measurements to the 61-pin fuel bundle assembly. The working fluid was p-

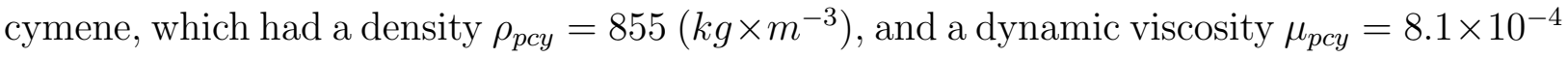
$(\mathrm{Pa} \times \mathrm{s})$. The p-cymene had nearly the same refractive index as acrylic $(n=1.49)$ at room temperature, and was used for the MIR technique to facilitate the PIV measurements in the edge, corner and interior sub-channels. The PIV system consisted of a $10 \mathrm{~W}$ continuous laser at a wavelength of $527 \mathrm{~nm}$ and two digital CMOS Phantom M310 cameras. The laser beam was adjusted by beam combination optics to form a 1-mm-thick laser sheet for the PIV measurements. For all experiments, the laser position was adjusted by using three motorized linear translation stages. The high-speed Phantom M310 cameras with a full resolution of $1280 \times 800$ pixels, a pixel size of $20 \times 20 \mu \mathrm{m}^{2}$ and 12-bit depth captured PIV images, and 

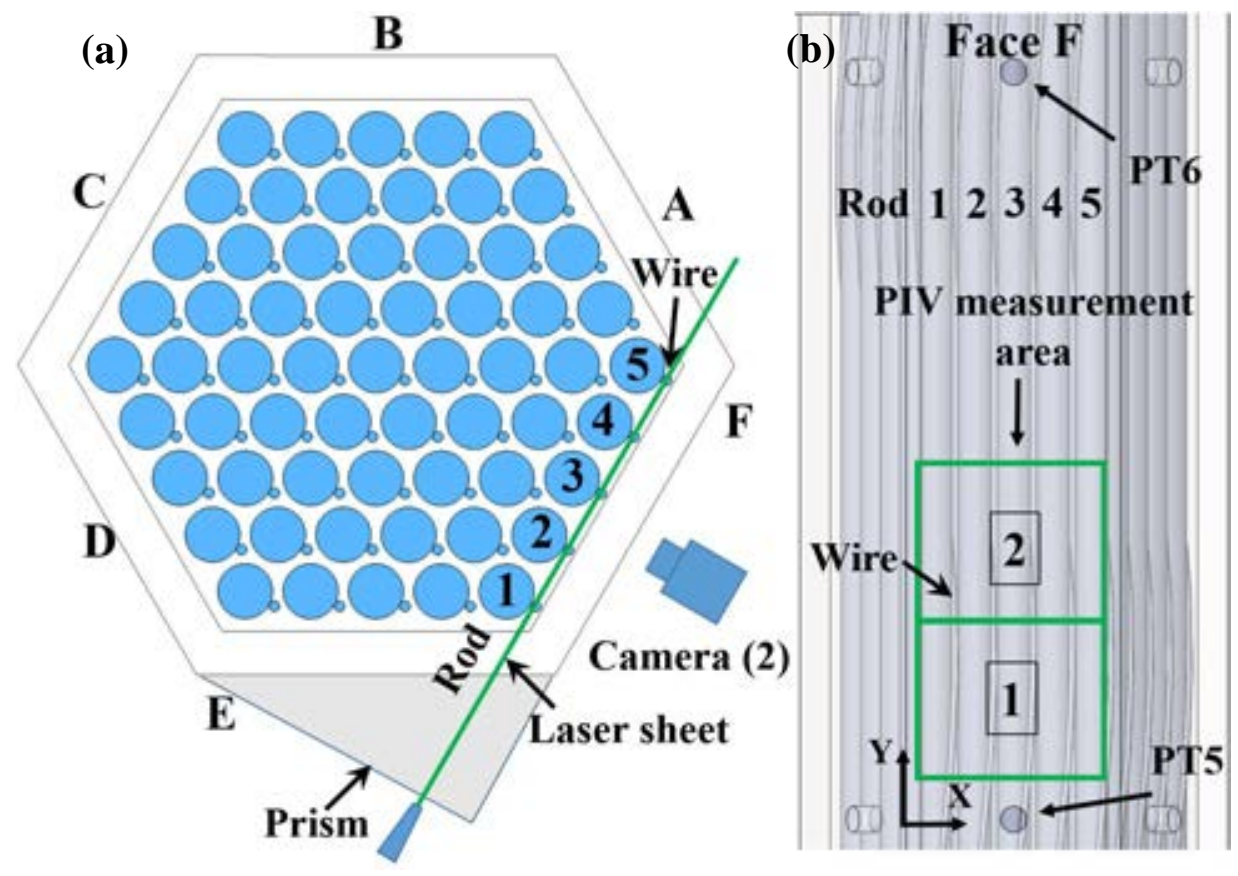

Figure 2: PIV experimental setup for the 61-pin helically wire-wrapped fuel bundle. (a) Top view and (b) front view of the PIV measurements in the near-wall region. Dynamic pressure transducers are mounted at locations PT5F and PT6F.

instantly stored them to the $12 \mathrm{~GB}$ high-speed internal RAM. Seeding particles were silvercoated hollow glass spheres with a mean diameter of $16 \mu \mathrm{m}$ and a density of $1.6 \mathrm{~g} \times \mathrm{cm}^{3}$, that yielded a strong greyscale intensity in the PIV images. The tracer particles were mixed in the primary tank. During the experiments, the fluid flow was driven from the primary tank, pumped into the fuel bundle via the inlet plenum, and returned to the tank. The flow, therefore, was circulated, and the tracers were present in the entire volume of the test section.

The 2D2C PIV measurement plane located in the region near the pins and wires, that were indicated by number $1-5$, and the enclosure wall as shown in figure 2 . The spatial gap between the pins, wires and enclosure wall of this near-wall region ranged from 0 to $3.8 \mathrm{~mm}$. In order to illuminate the axial-traversal $x y$-plane, the laser sheet was guided through a 30-60-90 triangular prism, which was attached on the exterior enclosure wall. In this measurement, we assigned $x$ and $y$ coordinates to represent the horizontal (traversal) and vertical (axial) directions, respectively. The origin of the coordinate system was at the bottom left corner of the region formed by the intersection of the laser sheet and enclosure wall, and at the elevation of $2.25 P_{t}$ from the test section entrance. In this paper, the PIV experimental results were obtained at a Reynolds number $R e=19,000$. The Reynolds 
number was defined by $R e=\left(U_{m} \times D_{h}\right) / \nu_{p c y}$, where $U_{m}$ was the mean bulk flow velocity, $D_{h}$ was the bundle hydraulic diameter, and $\nu_{p c y}$ was the kinematic viscosity of p-cymene. The velocity components corresponding to the $x$ and $y$ directions were $U$ and $V$ for the time-averaged velocity, and $u^{\prime}$ and $v^{\prime}$ for the fluctuating velocity, respectively.

During the PIV measurements, two cameras were mounted vertically, and their positions to the hexagonal fuel bundle were adjusted by three motorized linear translation stages. These cameras simultaneously recorded the flow images and their fields of views, noted by 1 and 2 in figure $\mathbf{Z ( b )}$, were adjoined to enlarge the measured areas. For each experimental run, each camera operated in a single-frame mode and captured a sequence of 8310 images of $1280 \times 800$ pixels with an image frame rate $f=2,800 \mathrm{~Hz}$. In order to compute the flow statistics, three experimental runs were performed. At the Reynolds number $R e=19,000$, the mean bulk flow velocity was $U_{m}=2.2(\mathrm{~m} / \mathrm{s})$ with $\nu_{p c y}=9.47 \times 10^{-7}\left(\mathrm{~m}^{2} / \mathrm{s}\right)$ estimated at 22 degrees Celsius. Within the operating frame rate $f$, we denoted the inter-frame time delay by $\Delta t=1 / f \approx 3.6 \times 10^{-4}$ (second) that yielded a maximum particle displacement of 15 pixels. The recording time for each experimental run covered about 2387 eddy turn-over times, where an eddy size could be reasonably determined as a radius of a circle subscribed within the gaps of a sub-channel formed by three neighboring pins and wires. With this assumption, the eddy size was estimated as $D_{e}=2.73(\mathrm{~mm})$ and the eddy turn-over time was defined by $D_{e} / U_{m}$. In another way, one could consider that the recording time was nearly 4 flow-through times, in which a flow-through time was defined as the time length the particles entered and exited the test section with the mean bulk velocity $U_{m}$. Provided that the recording times were long enough, and the numbers of image samples were large, results computed from the PIV measurements such as mean velocity, Reynolds stress components could statistically represent the flow characteristics of the fuel bundle.

All 12-bit depth PIV images captured by two cameras were processed by the advanced multi-pass, multi-grid PIV processing algorithms. These were based on robust phase correlation (RPC) algorithms that were implemented in the PRANA codes by Virginia Tech (Eckstein and Vlachos, 200),a, b). In the PIV processing, the initial and final interrogation windows were $64 \times 64$ pixels and $16 \times 16$ pixels, respectively. Particle displacements computed from the previous pass were used to shift the interrogation window in the next pass. All passes had a $50 \%$ window overlap, yielding the final spatial gap between two adjacent vectors of $0.72(\mathrm{~mm})$. Velocity vectors were calculated from the correlation map with a Gaussian peak fit for sub-pixel accuracy (Raffel et al., 2013). Inside each pass, statistical validations were performed to identify and replace erroneous vectors. A median filter (Westerweel, 1.994) was applied, and standard deviations of the neighboring vectors were used to filter out spurious vectors, and then blanks were filled by velocity interpolation. Veloc- 
ity vector fields obtained from two camera images were adjoined and interpolated to yield the larger field of $109 \times 176$ vectors without applying any smoothing process. For all the PIV processing of the experimental runs, the percentage of bad vectors, which was defined as the number of vectors failed the validation process in the final pass, and averaged over the number of PIV velocity fields, was less than 3\%. Performance and uncertainty of the PRANA RPC algorithm were accessed in Timmins et al. (2012), Wilson and Smith (2013), Charonko and Vlachos (2013), and Boomsma et al. (2016). The overall uncertainty in the PIV velocity measurements was estimated approximately 0.1 pixels yielding less than $3 \%$ of the mean axial velocity in the near-wall measurement plane.

\subsection{Fluctuating pressure measurement}

In accordance to the PIV measurements in the near-wall region, fluctuating pressure on the enclosure wall was measured by two dynamic pressure transducers Omega DPX101250 at 8,400 Hz. These two flush-mount pressure transducers, named PT5 and PT6, were installed on the face $F$, and located at the axial locations of $2.25 P_{t}$ and $3.0 P_{t}$, respectively. Those locations were upstream and downstream of the PIV measurement areas as indicated in figure 2(b). The dynamic pressure transducers transmitted voltage signals ranging from 0 to $5 \mathrm{~V}$ to the National Instrument DAQ system. The pressure transducers had a sensitivity of 168 $\mathrm{mV} / \mathrm{MPa}$, and an uncertainty of $\pm 0.5 \mathrm{MPa}$. In order to synchronize the image acquisitions and pressure recording, a pulse-width $20 \mu$ s signal was sent from a pulse generator to the cameras and the DAQ system.

\section{Results from PIV measurements in the near-wall region}

\subsection{Velocity field statistics}

A total of $N_{\max }=24,927$ instantaneous velocity vector fields acquired from three experimental runs could be used to compute the flow statistics. In this paragraph, convergences of the first-order and second-order flow statistics, such as mean velocity and Reynolds stresses, respectively, and two-point cross-correlations, depending on the number of PIV realizations are discussed. Two strategies were applied in order to justify the convergence of flow statistics. In the first strategy, we compared the PIV mean velocity and root-mean-square (r.m.s) fluctuating velocity profiles obtained along three horizontal lines, named $H_{1}\left(y_{1}=60\right) \mathrm{mm}$, $H_{2}\left(y_{2}=90\right) \mathrm{mm}$, and $H_{3}\left(y_{3}=120\right) \mathrm{mm}$, respectively. The first-order and second-order statistics profiles were computed by using $N_{1}=10,000, N_{2}=15,000, N_{3}=20,000$ and $N_{\max }$ velocity snapshots. Figure 3 illustrates the comparisons of the normalized mean axial velocity $V / U_{m}$ and r.m.s axial fluctuating velocity $v_{r m s}^{\prime} / U_{m}$ along the lines $H_{1}, H_{2}$, and $H_{3}$. In figure 

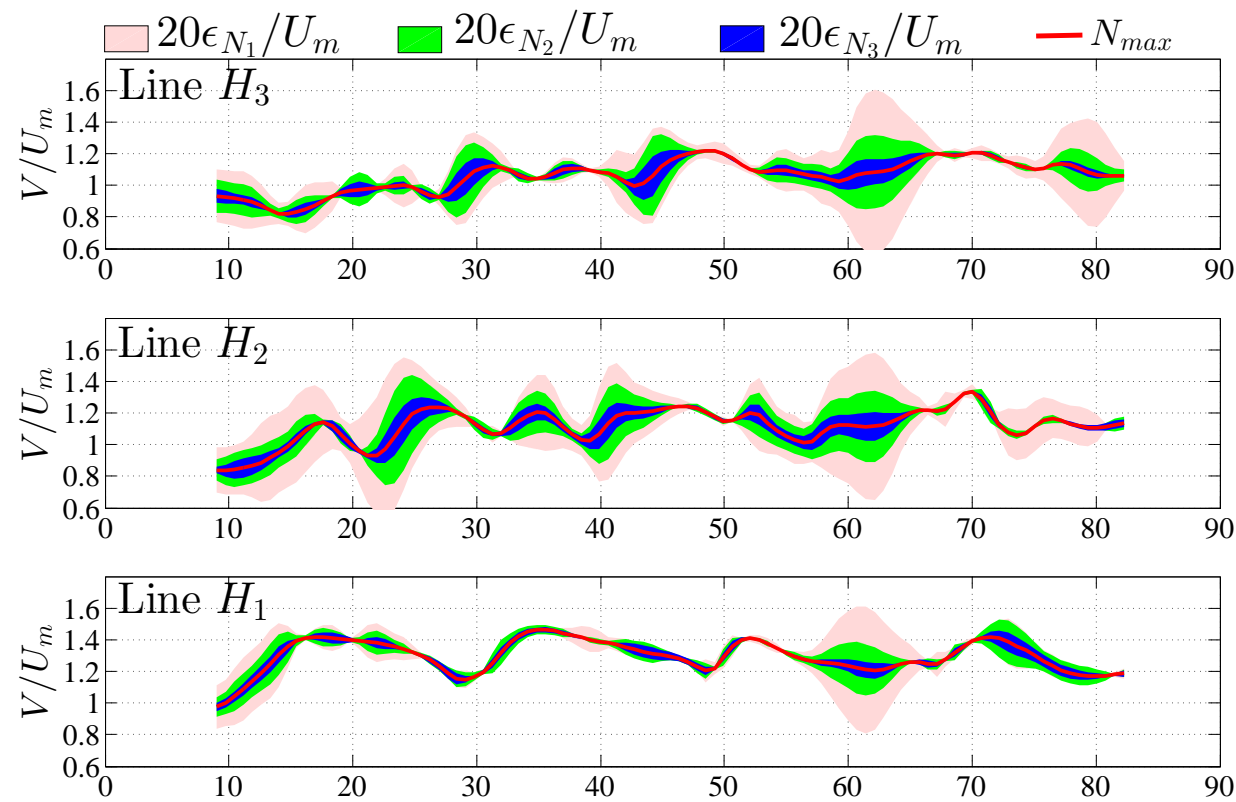

(a)
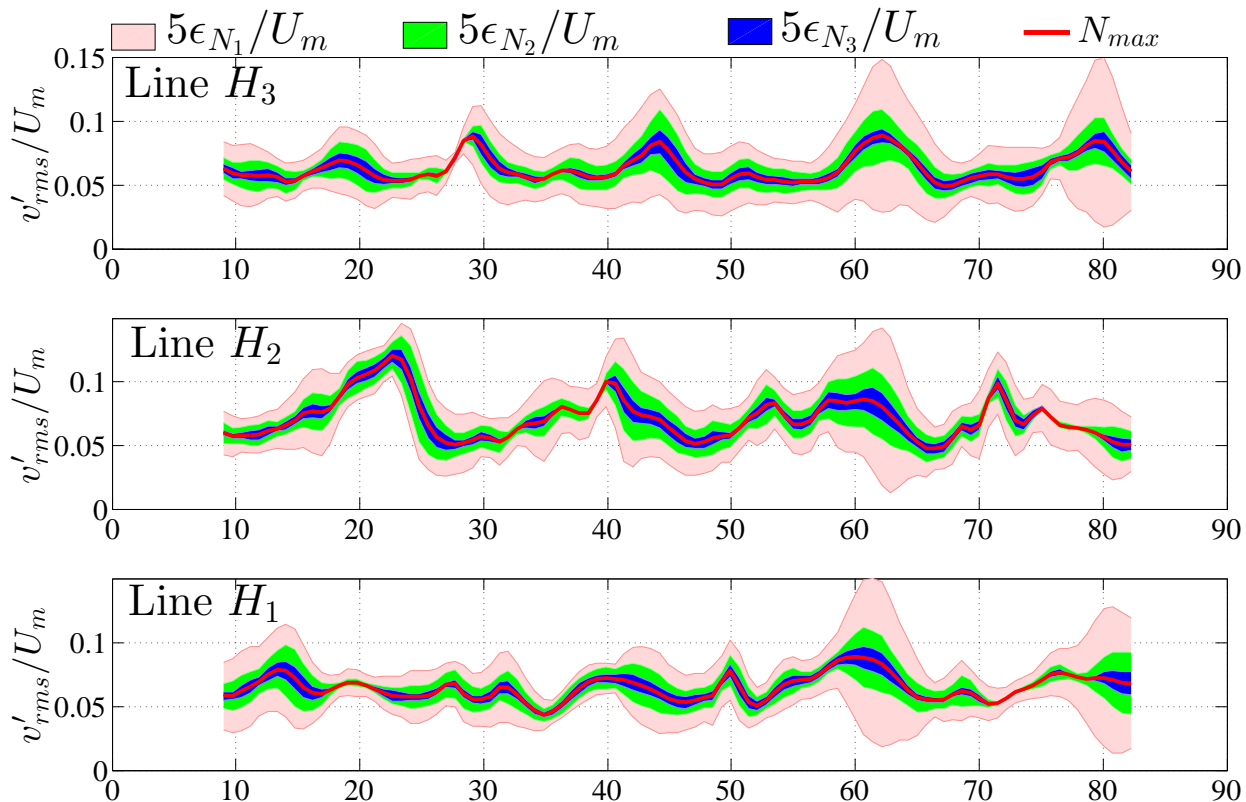

(b)

Horizontal axis, $\mathrm{X}(\mathrm{mm})$

Figure 3: PIV results obtained from the near-wall region for $R e=19,000$. (a) Normalized mean velocity $V / U_{m}$, and (b) normalized r.m.s fluctuating axial velocity $v_{r m s}^{\prime} / U_{m}$ computed along three lines $H_{1}\left(y_{1}=60\right)$ $\mathrm{mm}, H_{2}\left(y_{2}=90\right) \mathrm{mm}$, and $H_{3}\left(y_{3}=120\right) \mathrm{mm}$. Shaded colors show the normalized absolute differences $\epsilon_{N_{1-3}} / U_{m}$ between the statistics computed using $N_{1-3}$ samples and those using $N_{\max }$ snapshots. Note that to aid the visualization, shaded areas were amplified by the factors indicated in the legends. 
31, shaded colors illustrate $\epsilon_{N_{1-3}}$, which were defined as the absolute differences between the statistics computed by using $N_{1-3}$ snapshots and those by using the total $N_{\max }$ samples, respectively. Note that in figure [3, $\epsilon_{N_{1-3}}$ were normalized by $U_{m}$, yielding $\epsilon_{N_{1-3}} / U_{m}$, and to aid the visualization on the comparisons, the shaded areas representing the magnitudes of $\epsilon_{N_{1-3}} / U_{m}$ were amplified by factors $S_{1-3}$, yielding $S_{1-3} \times \epsilon_{N_{1-3}} / U_{m}$. The comparisons in figure [3] showed that as the numbers of samples used to compute the flow statistics increased from $N_{1}$ to $N_{3}$, the magnitudes of $\epsilon_{N_{1-3}} / U_{m}$ reduced from $3 \%$ to $0.6 \%$ for the normalized mean axial velocity $V / U_{m}$, and from $1.3 \%$ to $0.3 \%$ for the normalized r.m.s fluctuating velocity $v_{r m s}^{\prime} / U_{m}$. In the second strategy, following Rainer et al. (2016), we evaluated the convergence of two-point spatial cross-correlations computed by using various numbers of samples, i.e. $2,000,5,000$, and 8,310 velocity vector fields, taken from an experimental run. The reference points chosen to compute the two-point cross-correlations were $O_{1}\left(x_{1}, y_{1}\right)=(20,60) \mathrm{mm}$, $O_{2}\left(x_{2}, y_{2}\right)=(45,90) \mathrm{mm}$, and $O_{3}\left(x_{3}, y_{3}\right)=(70,120) \mathrm{mm}$. These points are located on the upward side, top side and downward side of the pins, respectively. Shapes of the two-point

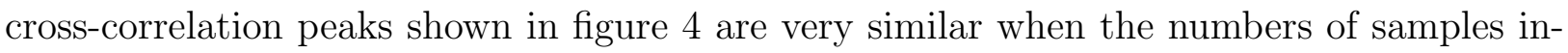
creased from 5,000 to 8,310. This indicates that the use of 8,310 velocity vector fields is sufficient to obtain the statistically converged two-point cross-correlations. As the conclusions, we used all the number of velocity vector fields collected from three experimental runs, i.e. $N_{\max }$, to compute the flow statistics, such as the mean velocity and Reynolds stresses, while the number of 8,310 instantaneous velocity vector fields obtained from one experimental run were used to compute the statistical values containing the temporal information, such as spatial cross-correlations, and spatial-temporal cross-correlations.

In this paragraph, we discuss the flow statistics computed from the PIV measurements. Figure 5(a) shows a snapshot of an instantaneous velocity vector field and vorticity contour obtained from the PIV measurements for $R e=19,000$. To visualize and interpret the findings, the obtained PIV results were overplotted on the 3D CAD model of the pins and wires of the fuel bundle. The instantaneous velocity vectors and corresponding vorticity contour showed that the near-wall flow followed the helical orientation of the wires, and the identified vortices had an elongated structure when moving in the narrow gaps between the pins and the enclosure wall. These observations are confirmed in the contour plots of the mean vorticity and velocity streamlines as shown in figure 5(b). In addition, one may find two flow regions where the vortical structures showed their appearances with alternating positive and negative strength. The first region was on the downward sides of the pins, and at the spatial locations where the wires were still in front of the pins. The second region was on the upward sides of the pins, and at the spatial locations where the wires started to move behind the pins. The mean velocity vectors and colormap of the axial velocity are displayed 

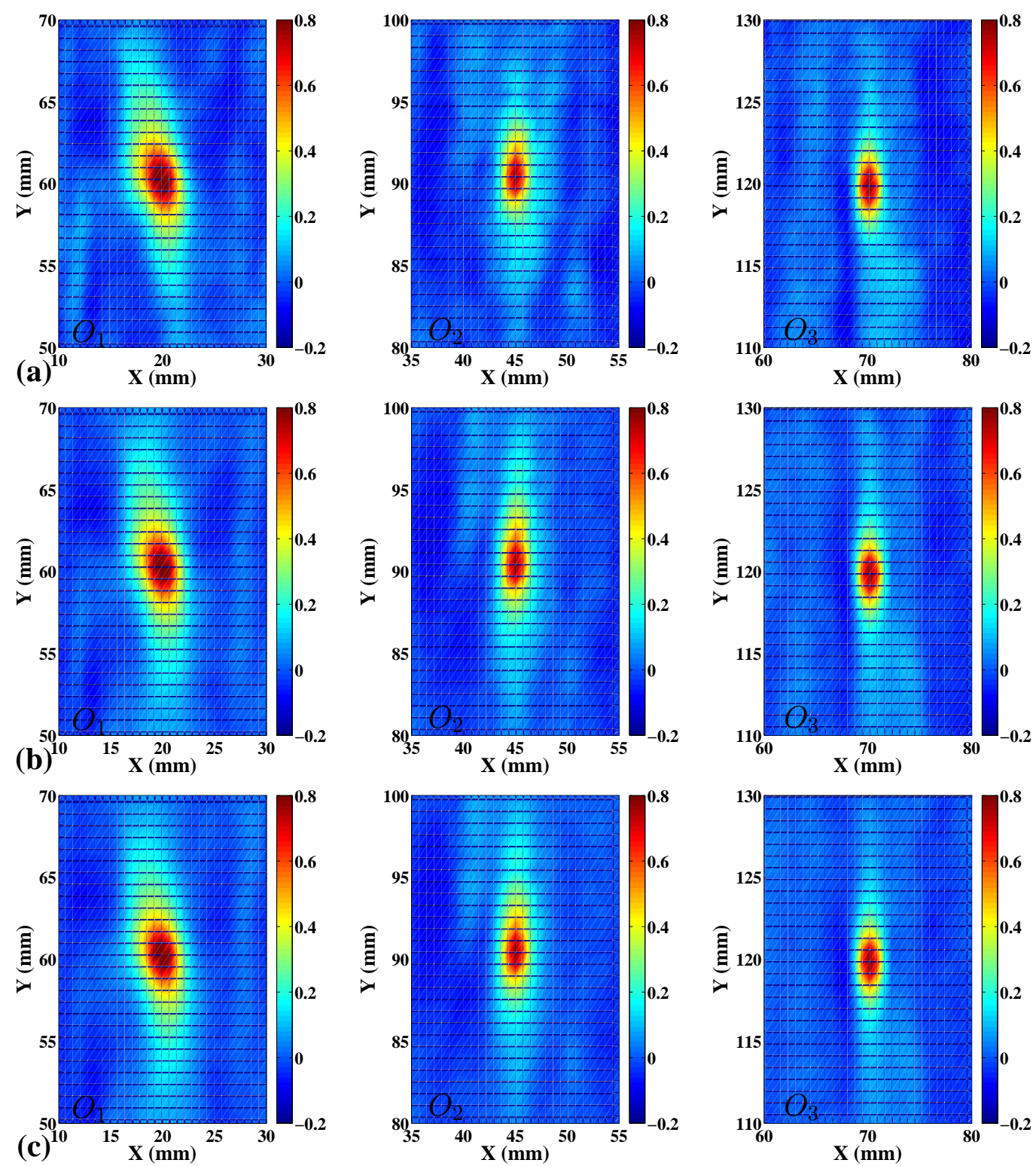

Figure 4: PIV results obtained from the near-wall region for $R e=19,000$. Two-point spatial cross-correlations of the fluctuating velocity $v^{\prime}$ computed by using (a) 2,000, (b) 5,000, and (c) 8,310 velocity vector fields. The reference points are $O_{1}(20,60), O_{2}(45,90)$, and $O_{3}(70,120) \mathrm{mm}$.

in figure 5 (c). It can be seen that the fluid flow traveled along the surfaces of the pins, wires and the enclosure inner wall, where the flow area gradually reduced and increased. Such changes of the flow area caused the flow accelerated and decelerated, respectively, when it passed through the upward and downward sides of the pins. Figure $5(\mathrm{~d})$ shows a color contour of the r.m.s fluctuating axial velocity $v_{r m s}^{\prime}$. Peaks of the contour map were found on the contact points of the wires and the upward sides of the pins, and also at the joining 
spots of the positive and negative vortices. It can be explained by the fact that after the near-wall flow was accelerated behind the downward sides of the pins, the change of the wire angle in helical direction caused a suddenly enlarged flow area that forced the flow to alter its direction inward to the interior sub-channels, and impinged onto the upward sides of the neighboring pins. The locations of $v_{r m s}^{\prime}$ peaks were found to coincide with the regions of high temperature (hot spots) and high fluctuating temperature that were observed in Ranjan et al. (201]) by using their direct numerical simulation (DNS). These authors discussed that the temperature was higher in the vicinity of the contacts between the wires, pins and wall due to flow confinement, which restricted outward heat transport. Moreover, the augmentation of temperature fluctuation was related to the accelerating and decelerating flows on the downward and upward sides of the pins.

The color contours of the mean velocity and r.m.s fluctuating velocity can be also used to interpret the statistics profiles plotted in figure B. In the comparisons of the profiles along line $H_{1}$, the mean velocity displayed local maxima at the locations of wires on the upward sides of the pins, where local minima were found for the r.m.s fluctuating axial velocity. On the other hands, local minima of $V / U_{m}$ along line $H_{1}$ were seen on the downward sides of the pins, where two side-peaks of $v_{r m s}^{\prime} / U_{m}$ were found. While the mean velocity profile along line $H_{1}$ was flat, those profiles along lines $H_{2}$ and $H_{3}$ showed more local maxima and minima. It can be explained by the presence of the wire that started to move in the helical direction and into the sub-channels, yielding a change in the flow direction and an increase of bypass flow going to the interior sub-channels. For the profiles along lines $\mathrm{H}_{2}$ and $\mathrm{H}_{3}$, the local maxima of $v_{r m s}^{\prime} / U_{m}$ were at the locations between the local maxima and minima of mean velocity, i.e. the highest slope between the local maxima and minima.

\subsection{Analysis of velocity-velocity and pressure-velocity cross-correlation}

In this section, to provide an insight to the turbulent flow characteristics in the near-wall region of the fuel bundle, we perform spectral analysis and compute coherent functions from the obtained PIV velocity vector fields. In addition, spatial-temporal cross-correlations of velocity-velocity, and those of pressure-velocity were computed from datasets of the PIV measurements at $2800 \mathrm{~Hz}$ and wall fluctuating pressure measurements at $8400 \mathrm{~Hz}$. Correlation functions may provide useful information on the shape and size, and roles of coherent structures (Dumas, 1990; Spalart, ए988; Romano, 1995). The velocity-velocity spatial-temporal correlations are defined as

$$
R_{u u}\left(\boldsymbol{x}_{r}, \boldsymbol{\eta}, \tau\right)=\frac{\left\langle u^{\prime}\left(\boldsymbol{x}_{r}, t\right) \times u^{\prime}\left(\boldsymbol{x}_{r}+\boldsymbol{\eta}, t+\tau\right)\right\rangle}{\sqrt{\left\langle u^{\prime 2}\left(\boldsymbol{x}_{r}, t\right)\right\rangle} \sqrt{\left\langle u^{\prime 2}\left(\boldsymbol{x}_{r}, t\right)\right\rangle}},
$$



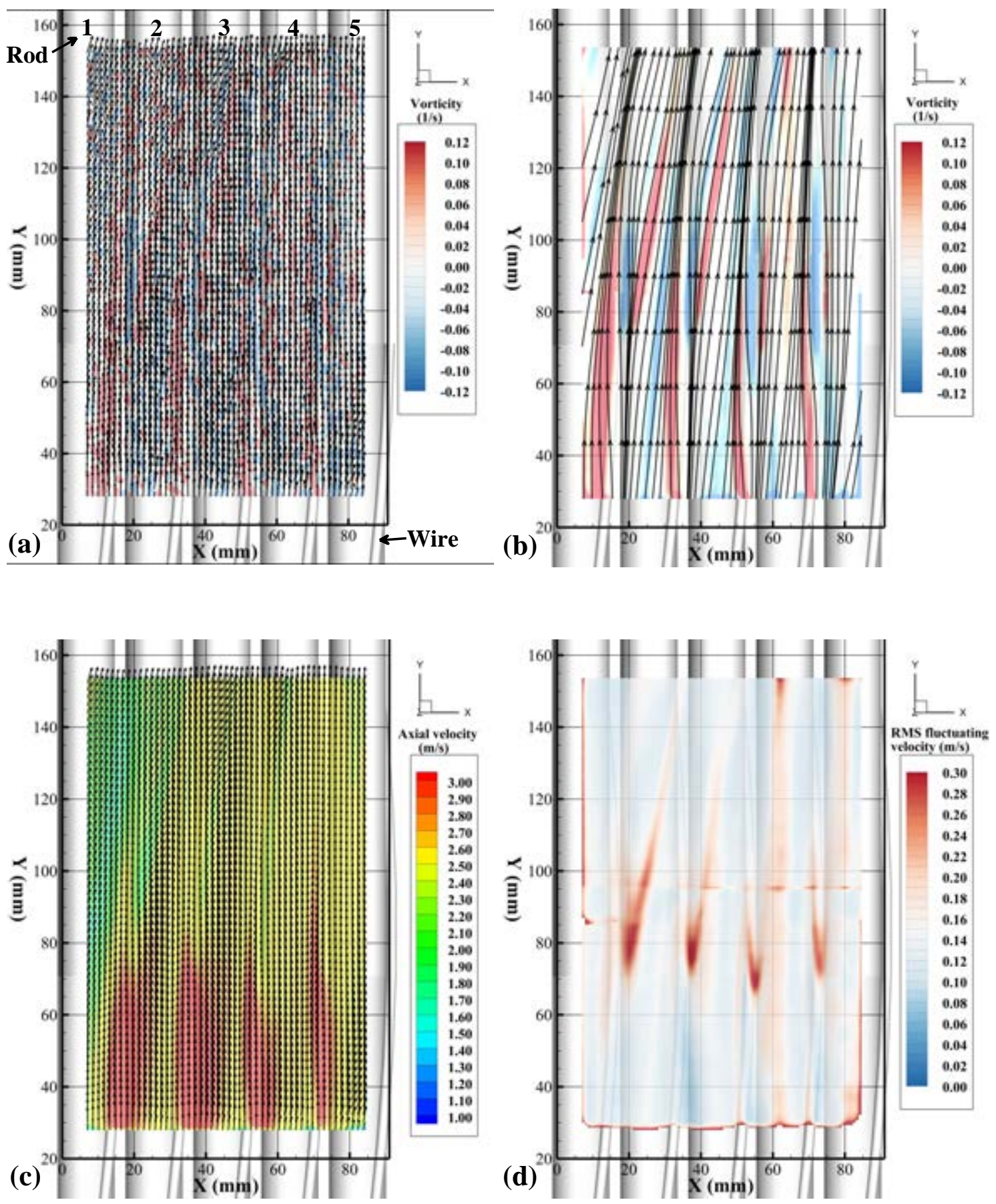

Figure 5: PIV results obtained from the near-wall region for $R e=19,000$. (a) Instantaneous velocity vectors and vorticity contour, (b) mean vorticity contour and velocity streamlines, (c) mean velocity vectors and axial velocity contour, and (d) a color contour of the r.m.s fluctuating axial velocity $v_{r m s}^{\prime}$. 

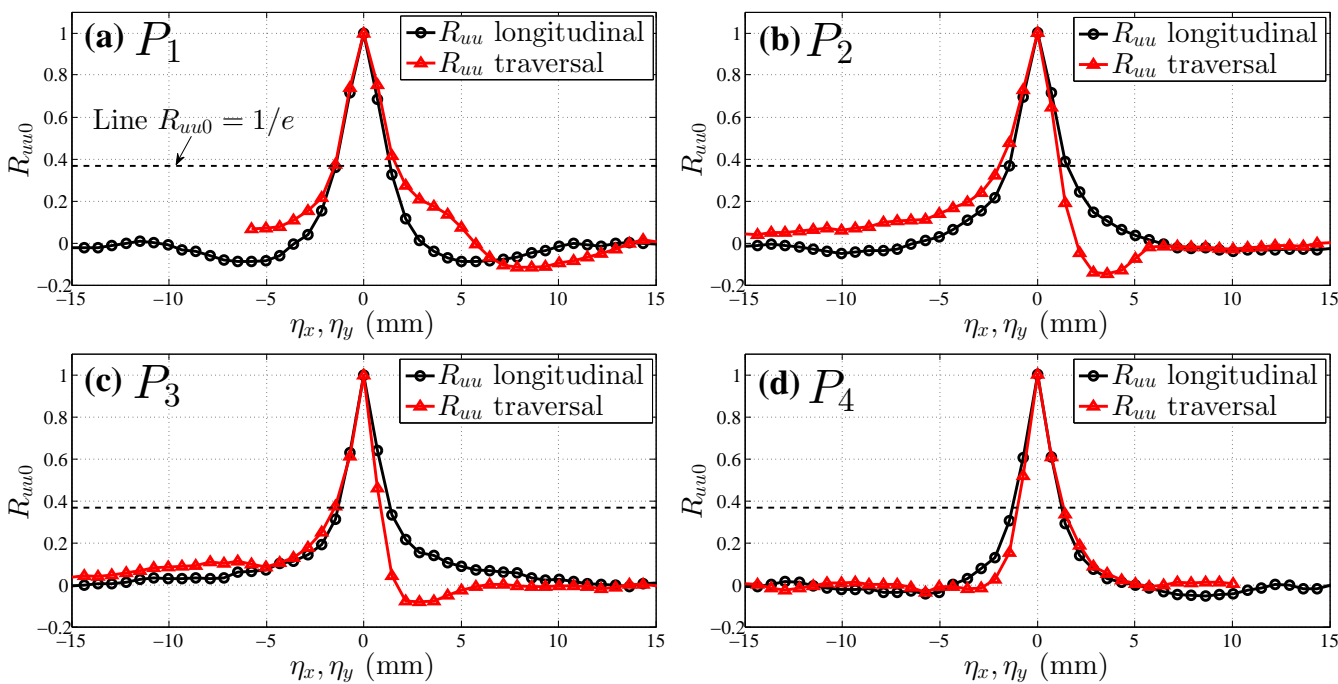

Figure 6: Spatial velocity-velocity cross-correlations $R_{u u 0}$ computed at 4 referenced points along the longitudinal and traversal directions. $R_{u u 0}$ at points (a) $P_{1}(12.7,60)$, (b) $P_{2}(35.7,60)$, (c) $P_{3}(54.4,60)$, and (d) $P_{4}(74.5,60) \mathrm{mm}$.
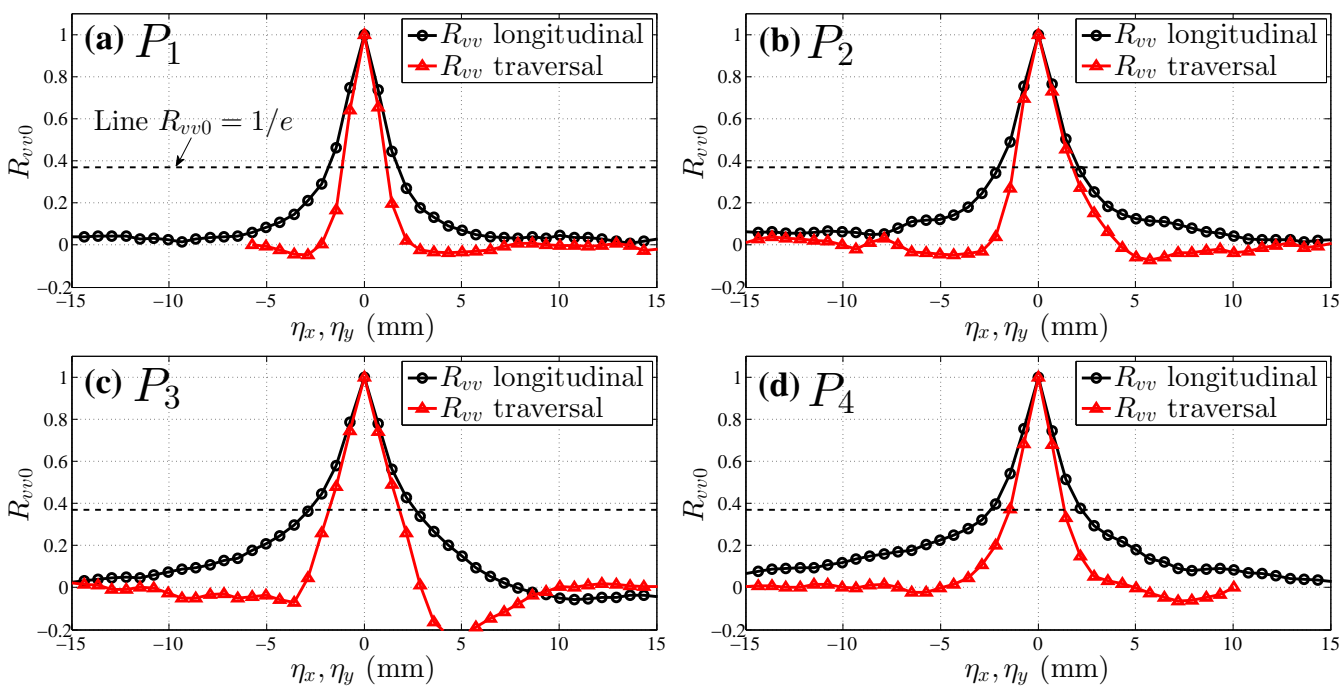

Figure 7: Spatial velocity-velocity cross-correlations $R_{v v 0}$ computed at 4 referenced points along the longitudinal and traversal directions. $R_{v v 0}$ at points (a) $P_{1}(12.7,60)$, (b) $P_{2}(35.7,60)$, (c) $P_{3}(54.4,60)$, and (d) $P_{4}(74.5,60) \mathrm{mm}$.

$$
R_{v v}\left(\boldsymbol{x}_{r}, \boldsymbol{\eta}, \tau\right)=\frac{\left\langle v^{\prime}\left(\boldsymbol{x}_{r}, t\right) \times v^{\prime}\left(\boldsymbol{x}_{r}+\boldsymbol{\eta}, t+\tau\right)\right\rangle}{\sqrt{\left\langle v^{\prime 2}\left(\boldsymbol{x}_{r}, t\right)\right\rangle} \sqrt{\left\langle v^{\prime 2}\left(\boldsymbol{x}_{r}, t\right)\right\rangle}},
$$

where $\langle$.$\rangle is the ensemble-averaged operator, u^{\prime}$ and $v^{\prime}$ are the fluctuating velocity components, $\tau$ is a time delay, $\mathbf{x}_{r}$ stands for the spatial locations of the reference points, and $\boldsymbol{\eta}$ is the spatial separation between two point measurements. In the equations (四) and (च), 
a special case when $\tau=0$ allows the calculations of spatial correlation length scales, i.e. $R_{u u 0}=R_{u u}\left(\boldsymbol{x}_{r}, \boldsymbol{\eta}, 0\right)$ and $R_{v v 0}=R_{v v}\left(\boldsymbol{x}_{r}, \boldsymbol{\eta}, 0\right)$, in a fixed reference time as a function of separation $\boldsymbol{\eta}$ (Kerhervé and Fitzpatrick, 2001). These correlations indicate the spatial extent of correlated regions (Jones et al, 1973). From the correlation length scales, the integral length scales are defined as

$$
\begin{aligned}
L_{x} & =\int_{0}^{\infty} R_{u u}\left(\boldsymbol{x}_{r}, \boldsymbol{\eta}, 0\right) d \eta, \\
L_{y} & =\int_{0}^{\infty} R_{v v}\left(\boldsymbol{x}_{r}, \boldsymbol{\eta}, 0\right) d \eta .
\end{aligned}
$$

The integral length scales $L_{x}$ and $L_{y}$ can be interpreted as the characteristic length scales of the energy containing turbulent eddies. In this study, instead of integrating the equations (B) and (甘), the integral length scales $L_{x}$ and $L_{y}$ were estimated by finding the separation length where $R_{u u 0}$ and $R_{v v 0}$ reached the value of $1 / e \approx 0.37$ (Romano, 1995); Falchi and Romano, 20(1)! .

Figures $\mathbf{6}$ and $\mathbb{0}$ show the spatial velocity-velocity cross-correlations calculated from the PIV velocity vector fields. Four referenced points $P_{1}, P_{2}, P_{3}$ and $P_{4}$ had the spatial coordinates of $\left(x_{1-4}, y_{1-4}\right)=(12.7,60),(35.7,60),(54.4,60)$ and $(74.5,60) \mathrm{mm}$, respectively. The spatial cross-correlations $R_{u u 0}$ and $R_{v v 0}$ were calculated along the longitudinal and traversal directions. The abscissa shown in figures $\mathbb{6}$ and $\square$ represent the separation lengths from the referenced points to others along the according directions. As the normalized calculations were used, the correlations $R_{u u 0}$ and $R_{v v 0}$ had the unity values at $\eta=0$, and decreased with an increase of separation distance. It can be seen in figure 6 that the $R_{u u 0}$ traversal correlations were smaller than the longitudinal correlations with the positive separation for points $P_{2}$ and $P_{3}$. While the $R_{u u 0}$ longitudinal and traversal correlations were equal in the positive separation at point $P_{4}$, these correlations seemed to alternate each other at point $P_{1}$. In figure $\square$, the spatial correlations $R_{v v 0}$ calculated at points $P_{1}-P_{4}$ showed the same trend that for both positive and negative separations, the longitudinal correlations were greater than the traversal correlations. As derived from the spatial correlations, the integral length scale $L_{y}$ was found to be greater than $L_{x}$ especially in the longitudinal direction, which was the dominant flow path. Specifically, along the longitudinal direction, the integral length scale $L_{x}$ ranged from $1.2(\mathrm{~mm})$ to $1.5(\mathrm{~mm})$, while the estimated $L_{y}$ was greater and ranged from $1.7(\mathrm{~mm})$ to $2.8(\mathrm{~mm})$. Along the traversal direction, the estimated $L_{x}$ varied from 0.9 $(\mathrm{mm})$ to $1.7(\mathrm{~mm})$, while $L_{y}$ varied from $1.1(\mathrm{~mm})$ to $1.8(\mathrm{~mm})$. While the maximal integral length scales were found at points $P_{3}$ and $P_{4}$, the length scales determined at points $P_{1}$ and $P_{2}$ were smaller. It should be noted that the correlations also showed negative minima, and a periodic alternating positive and negative peaks at larger separations. An explanation for 

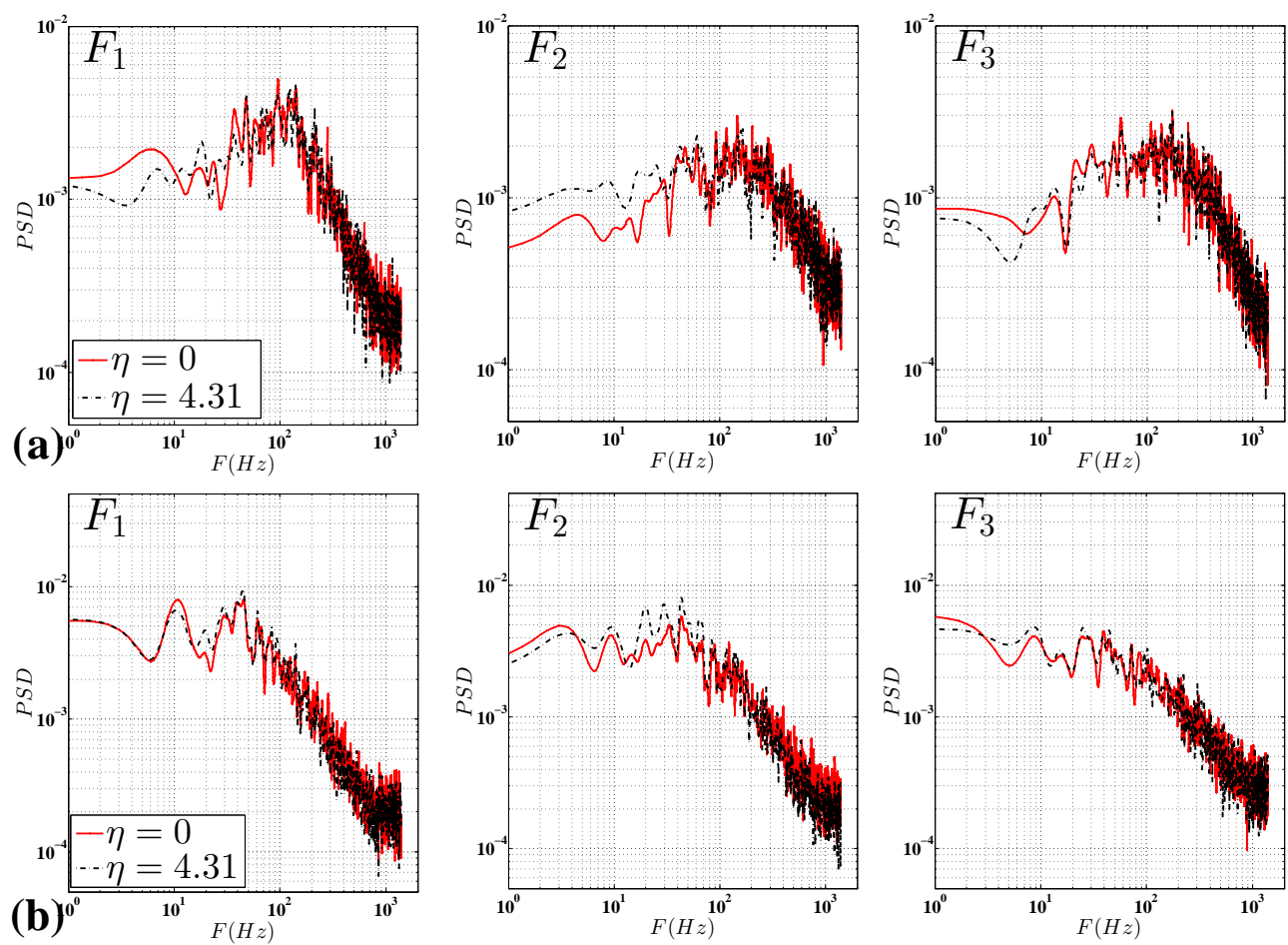

Figure 8: Spectral analysis of the fluctuating velocity (a) $u^{\prime}$, and (b) $v^{\prime}$ obtained at points $F_{1}(20,120)$, $F_{2}(45,120)$, and $F_{3}(70,120)(\mathrm{mm})$, and other points with a separation of $\eta=4.31(\mathrm{~mm})$.

the above findings could be the highly correlated regions for large distances in the longitudinal and traversal directions. Such correlated regions were indicative of the streamwise convection of travelling vortices.

The calculations of spatial cross-correlations $R_{u u 0}$ and $R_{v v 0}$ allowed the estimates of the integral length scales, which were the large scale coherent structures of the studied turbulent flows. However, no information on the frequency of these energy containing structures was identified. In this paragraph, we performed spectral analysis to the fluctuating velocity $u^{\prime}$ and $v^{\prime}$ components at three points $F_{1}, F_{2}$ and $F_{3}$, whose coordinates were $\left(x_{1-3}, y_{1-3}\right)=(20,120)$, $(45,120)$, and $(70,120) \mathrm{mm}$, respectively. These points were located on the upward side, the top side and the downward side of the pins in the near-wall PIV measurement plane. Coherent functions computed between the points $F_{1}, F_{2}, F_{3}$ and other points corresponding to various separation lengths along the axial direction, i.e. points with coordinates $\left(x_{1-3}, y_{1-3}+\eta\right)$, were presented. For the given points $A$ and $B$, the squared coherent function between these points is defined as

$$
C_{A B}(f)=\frac{\left|P_{A B}(f)\right|^{2}}{P_{A A}(f) P_{B B}(f)}
$$

where $P_{A A}(f), P_{B B}(f)$ and $P_{A B}(f)$ are power spectral densities (PSD), and the cross power 

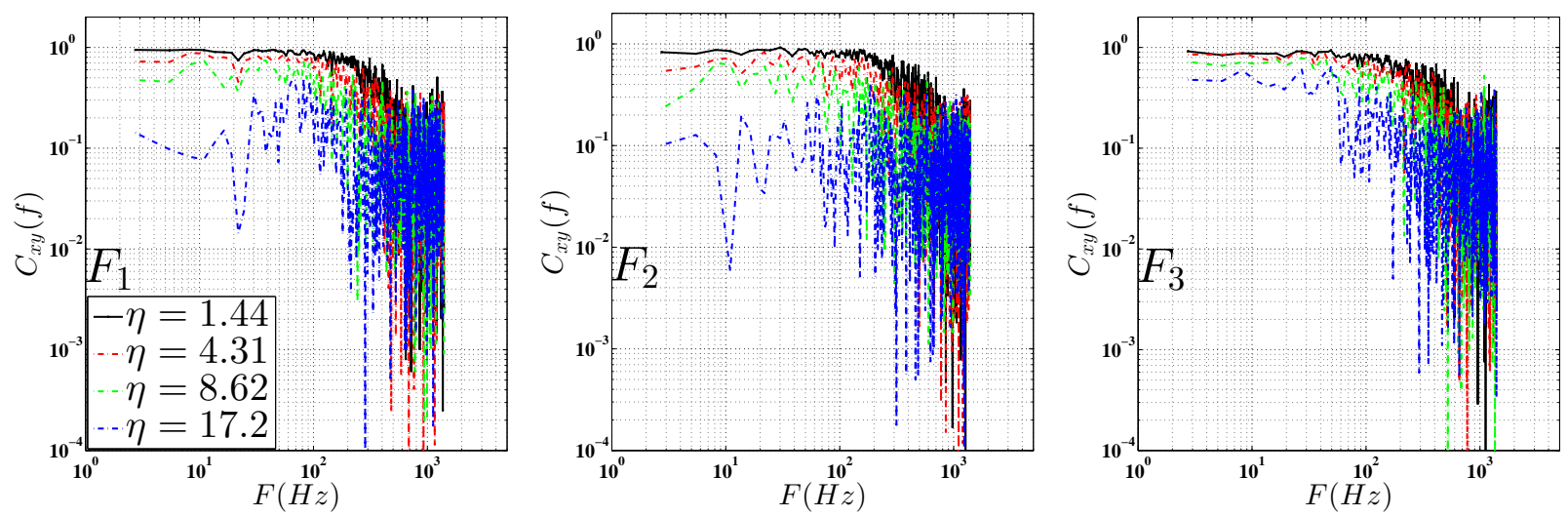

Figure 9: Squared coherent functions versus frequency computed at points $F_{1}(20,120), F_{2}(45,120)$, and $F_{3}(70,120) \mathrm{mm}$ with various separations of $\eta=1.44,4.31,8.62$, and $17.2 \mathrm{~mm}$.

spectral density of $A$ and $B$, respectively. The coherent function represents the portion of mean square signal at point $B$ and at a frequency $f$ that is accounted for by the signal at point $A$ (Romano, 1995).

In figure $\mathbf{8}$, the power spectral densities (PSD) computed for the PIV fluctuating velocity $u^{\prime}$ and $v^{\prime}$ obtained at points $F_{1}, F_{2}$ and $F_{3}$ are illustrated by curves with $\eta=0$. Spectral analysis of points with the separation length of $\eta=4.31(\mathrm{~mm})$, i.e. about two times of the integral length scale, is also overplotted. The PSD were estimated using Welch's periodogram method (Welch, 1967). The number of samples in each segment length was chosen to cover about 100 eddy-turn over times, and had 50\% overlapping sample. Peaks in the spectra representing the vortex shedding covered a wide frequency range, and no significantly dominant frequency was detected. It is seen that the spectra computed at points with $\eta=0$ and $\eta=4.31$ showed similar patterns. The analysis of coherent function was then applied to study the signal relations between different points versus the frequency. Figure $\mathbb{Q}$ shows the squared coherent functions computed at points $F_{1}, F_{2}$ and $F_{3}$ with various separation lengths $\eta=1.44,4.31,8.62$ and $17.2 \mathrm{~mm}$, i.e. approximately $0.5,2,4$ and 8 times of the integral length scales. In all cases, for small separations, the coherent functions remained close to unity at low frequencies up to $100 \mathrm{~Hz}$, indicating that the low-frequency content at the correspondingly calculated points was linearly related. For the same findings, Romano (199.5) explained that this is because the low frequencies, related to the mean flow interactions, are involved in the energy transfer process between the chosen points. However, as the separation increased, the linearity is rapidly lost, and the coherent function reduces.

The evolution of the spatial-temporal velocity-velocity cross-correlation $R_{v v}$ along the longitudinal direction computed for the referenced points $F_{1}, F_{2}$ and $F_{3}$ is illustrated in 

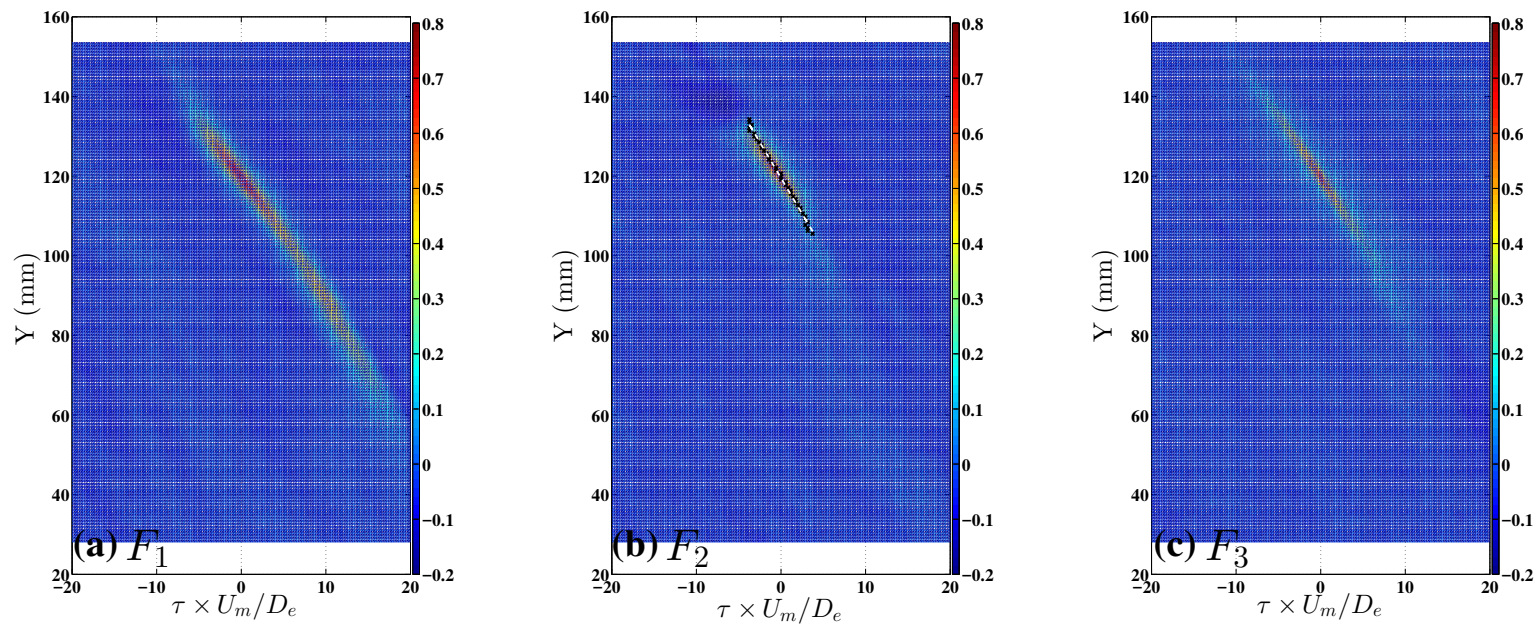

Figure 10: Spatial-temporal velocity-velocity cross-correlations $R_{v v}$ computed along the longitudinal direction at points (a) $F_{1}(20,120)$, (b) $F_{2}(45,120)$, and (c) $F_{3}(70,120) \mathrm{mm}$.

figure $\mathbf{1 0}$. In these color contours, the horizontal axis is the time delay normalized by the eddy turn-over time $D_{e} / U_{m}$, while the vertical axis represents the axial locations of points along the longitudinal direction, i.e. $\left(x_{1-3}, y_{1-3}+\eta\right)$ with $y_{1-3}=120(\mathrm{~mm})$, that contribute into the calculations of cross-correlation shown in equation (घ). The cross-correlation values achieved unity at the locations of referenced points $(x, y)=\left(x_{1-3}, y_{1-3}\right)$ and time delay $\tau=0$, and then reduced when the separation increased. In addition, as the separation length enlarged, the corresponding correlations reached their maxima values with greater normalized time delays. It is interesting to find that the shape of correlation peaks obtained at each referenced point was different. Because point $F_{1}$ located on the upward side of the pin, where the elongated vortices from the upstream impinged on the pin's surface and broke into smaller sizes, the turbulent velocity at this point was strongly correlated to that at other points along the longitudinal direction. As a result, correlation peak at point $F_{1}$ had the widest and longest shape. In contrast, correlation map at point $F_{3}$ had the thinnest shape as this point located on the downward side of the pin, where the vortices at smaller sizes probably started to move into the interior sub-channels. The distributions of spatial-temporal correlation peaks shown in figure $\mathbf{0}$ can be used to estimate the convection velocity $U_{c}$ by fitting a straight line onto the correlation peaks, and then evaluating the slope of the line (Romano, 1995; Hudy et al., 2007). In figure [0(b), the maximal correlation peaks detected at various separations and time delays are indicated by " $\mathrm{X}$ " markers, while the linear least square fitting is shown by the straight line (similarly done for points $F_{1}$ and $F_{3}$ but not 


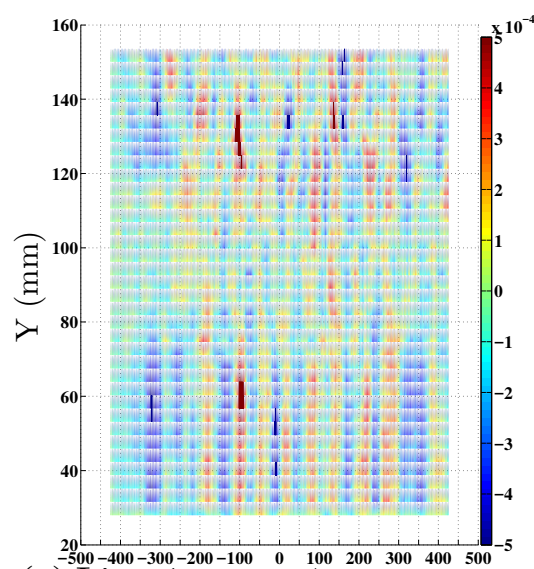

(a) Line $1 \tau \times U_{m} / D_{e}^{200-400-300-200-100}$

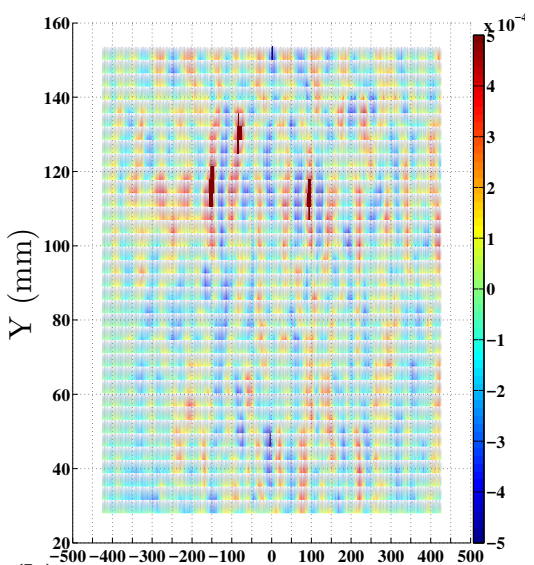

(b) Line $2 \stackrel{-500-400-300-200-100}{\tau} \times U_{m}^{0} / D_{e}^{100}$

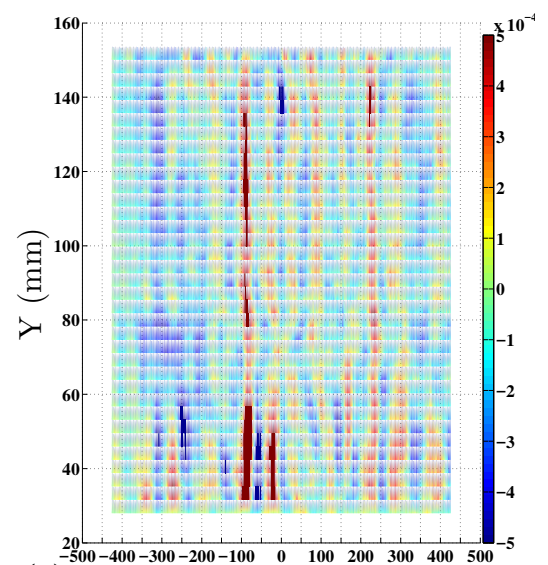

(c) $\operatorname{Line}^{-500-400-300-200-100} \tau \times U_{m}^{0} / D_{e}^{100}$

Figure 11: Spatial-temporal cross-correlation $R_{p v}(\boldsymbol{x}, \tau)$ calculated from the wall fluctuating pressure PT5F and PIV fluctuating velocity $v^{\prime}$ along three axial lines in the near-wall plane. $R_{p v}(\boldsymbol{x}, \tau)$ at $(a)$ line 1 with $L_{1}\left(x_{L_{1}}=35\right)$, (b) line 2 with $L_{2}\left(x_{L_{2}}=45\right)$, and (c) line 3 with $L_{3}\left(x_{L_{3}}=54\right) \mathrm{mm}$.

shown here). As discussed in Jones et al. (1973), the convection velocity was different from the local mean velocity and statistically depends on the spatial regions. In this paper, the convection velocity $U_{c}=0.93(\mathrm{~m} / \mathrm{s})$ was averaged from the values calculated at points $F_{1}$, $F_{2}$ and $F_{3}$ to represent the moving speeds of small and large scale structures.

In this paragraph, we discuss on the spatial-temporal cross-correlations between the wall fluctuating pressure and fluctuating velocity fields, i.e. $R_{p v}(\boldsymbol{x}, \tau)$, defined by

$$
R_{p v}(\boldsymbol{x}, \tau)=\frac{\left\langle p(t+\tau) \times v^{\prime}(\boldsymbol{x}, \tau)\right\rangle}{1 / 2 \rho_{p c y} U_{m}^{3}}
$$

where $p(t)$ is the wall fluctuating pressure measured by the dynamic pressure transducer, $v^{\prime}(\boldsymbol{x}, t)$ is the fluctuating axial velocity vector obtained from the PIV measurements in the near-wall plane, and $\tau$ is the time delay. Many researchers, for example Kiya and Sasaki (1983), Cherry et al. (1984), Lee and Sung (2002), and Ruiz et al. (2010), showed that the wall fluctuating pressure was associated with flow structures with large spatial and temporal coherent. In this work, the wall fluctuating pressure was obtained from PT5F, which located about $30(\mathrm{~mm})$ upstream of the first grid points in the PIV measurement plane. As noted by Ruiz et al (2010) and similarly seen in this paper, the contribution of wall fluctuating pressure to the cross-correlation was found to decrease when the distances between the measurement points of pressure and velocity increased. 
Figure [1] illustrates the color maps of normalized spatial-temporal cross-correlations $R_{p v}(\boldsymbol{x}, \tau)$ computed along three axial lines $L_{1}, L_{2}$ and $L_{3}$. The referenced pressure PT5F located at $\left(x_{P T 5 F}, y_{P T 5 F}\right)=(44.4,0)(\mathrm{mm})$, and three axial lines $L_{1}, L_{2}$ and $L_{3}$ were $x_{L_{1}}=$ $35, x_{L_{2}}=44.4$, and $x_{L_{3}}=54(\mathrm{~mm})$, respectively. These lines were located on the upward side, top side and downward side of the central pin. It is seen from the color maps of $R_{p v}(\boldsymbol{x}, \tau)$ that the cross-correlation patterns were different at various calculation points. The cross-correlation peaks appeared in both negative and positive time delays. Overall, the color contours showed a strong correlation between the wall fluctuating pressure and flow structures. Relations among the coherent flow structures and associated events, such as single-point and multi-point measurements of pressures, wall shear stress, velocity, etc., were discussed in many previous studies (Ukeiley and Murray, 2010.5; Hudy et al., 20107; Durgesh and Naughton, 2010; Ruiz et al., 2010; Nguyen et al., 2010). In addition, in these studies, the strong correlations between the coherent structures and associated events were employed to develop the simplified, reduced-order models to estimate the flow fields. It is widely known that vortical structures usually have complex shapes, and dynamically evolve in turbulent flows. In the current study, the pressure-velocity cross-correlations revealed an evident that footprints of the coherent structures being captured by the correlated pressure signals when these large-scale structures evolved in the near-wall region. In figure $\mathbb{D}$, the contour maps of $R_{p v}(\boldsymbol{x}, \tau)$ computed along the lines $L_{1}$ and $L_{3}$ showed periodic patterns of the vortex shedding in the lower region ranging from $y=30$ to $70(\mathrm{~mm})$, while the $R_{p v}(\boldsymbol{x}, \tau)$ contour calculated along the line $L_{2}$ revealed those patterns in the upper region ranging from $y=100$ to $140(\mathrm{~mm})$. These observations can be explained by the spatial locations of these three axial lines in associated with the instantaneous and mean vorticity structures shown in figure 5. Indeed, the lines $L_{1}$ and $L_{3}$ were located in the flow regions with high populations of streamwise vortices in the range from $y=30$ to $70(\mathrm{~mm})$, while the population of instantaneous vortices passing through the line $L_{2}$ was dominant in the region from $y=100$ to 140 (mm).

\subsection{POD analysis to the vorticity fields}

The uses of two-point spatial-temporal cross-correlations discussed in the previous section allowed us to identify various important parameters for turbulence studies. However, the spatial and temporal evolution of coherent structures in the full-field of measurement plane could not be directly derived from the two-point correlations. In this section, we apply the POD analysis to the snapshots of vorticity fields computed from the PIV instantaneous velocity vectors in order to reveal the most dominant flow structures, which play an important role in the flow dynamics and heat transfer of the fuel bundle. As pointed out in 

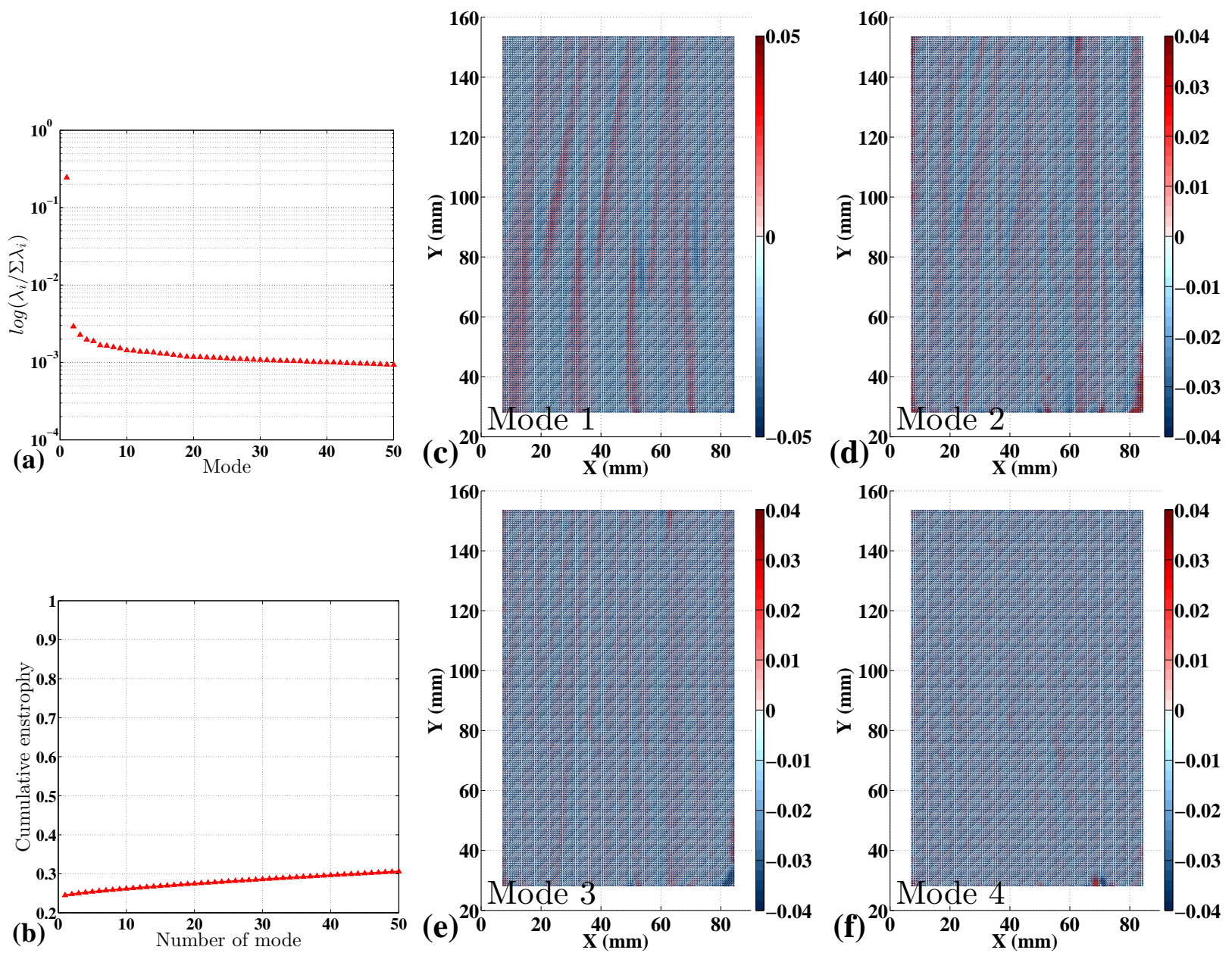

Figure 12: POD analysis of the PIV vorticity fields. (a) Enstrophy spectra, (b) cumulative enstrophy, and $(c-f)$ POD vorticity modes $1-4$.

Perry and Chong (11993), performing the POD analysis to the velocity fields may not be ideally appropriate to coherent identification since the derived structures will depend on the observer velocity. Moreover, according to the definition of coherent structure in Hussain (11986), and discussions in Kostas et al. (2005), Liberzon et al. (2005), and Gurka et al. (2006), POD analysis of the vorticity fields could efficiently reveal the coherent structures as vorticity is a Galiean invariant quantity. Detailed descriptions of the direct and snapshot POD techniques can be reviewed in Berkooz et al. (1993), Holmes et al. (1998), and Sirovich and Kirby (1987). A brief review of the snapshot POD to the vorticity fields is described here. First, a vorticity correlation matrix is defined as

$$
C_{i j}=\frac{1}{N} \int \boldsymbol{\omega}\left(\boldsymbol{x}, t_{i}\right) \cdot \boldsymbol{\omega}\left(\boldsymbol{x}, t_{j}\right) d \boldsymbol{x},
$$


where $N$ is the number of snapshots, and $\boldsymbol{\omega}(\boldsymbol{x}, t)$ stands for the PIV vorticity realizations. Next, let us define $\alpha_{k i}$ as

$$
\alpha_{k i}=\frac{v_{i}^{k}}{\sqrt{N \sum_{m=1}^{N} \sum_{r=1}^{N} v_{m}^{k} v_{r}^{k} C_{m r}}},
$$

where $v_{i}^{k}$ is the $i$ th element of the eigenvector $v^{k}$ corresponding to $\lambda_{k}$. The POD basis functions $\boldsymbol{\Psi}(\boldsymbol{x})$ and POD temporal coefficients $\zeta_{k}(t)$ are calculated as following

$$
\begin{aligned}
\boldsymbol{\Psi}_{k}(\boldsymbol{x}) & =\sum_{i=1}^{N} \alpha_{k i} \boldsymbol{\omega}\left(\boldsymbol{x}, t_{i}\right), \\
\zeta_{k}\left(t_{j}\right) & =N \sum_{i=1}^{N} \alpha_{k i} C_{i t_{j}} .
\end{aligned}
$$

As the POD analysis is applied to the vorticity fields, the eigenvalue $\lambda$ associated with each POD mode is proportional to the flow enstrophy whose density is defined as $\|\omega\|^{2}$. The decomposition yields statistically dominant flow structures in the few lowest-order POD modes that contribute a considerable proportion of the total flow enstrophy. These captured large-scale structures play important role in fluid dynamics and heat transfer. The POD analysis was applied to 8310 vorticity fields that were de-sampled from the total number of samples $N_{\max }$. This de-sampling yielded a greater inter-frame time delay, i.e. $3 \Delta t$; while a longer flow duration, i.e. more than 7100 eddy turn-over times, was covered by the dataset. Therefore, the vorticity snapshots are statistically independent, and the computed POD modes represent statistically dominant flow structures.

Figure 12 (a) shows the enstrophy spectra from the POD vorticity decomposition. The first POD mode, which was approximately equal to the averaged vorticity field (see figure 5(b) and figure ए2(b) for the comparison), captured $24 \%$ of the total flow enstrophy. The enstrophy level captured by each of other low-order POD vorticity modes was less than $1 \%$, yielding a slow convergence of the cumulative enstrophy. As similarly found in Kostas et al (2010.5) and Gurka et al (20106), the POD vorticity decomposition yielded low values of enstrophy containing in each mode compared to those values of kinetic energy in each mode from the POD velocity decomposition (results not shown here). In the current study, the fact that each of the first few POD modes contained a small enstrophy fraction and a slow convergence of cumulative enstrophy indicates that near-wall flow region is highly turbulent, and the flow energy is distributed over many small-scale structures (Kostas et al, 20105; Nguyen et al., 2012). Figure 12(c-f) illustrate the contours of the POD modes 2, 3 and 4 from the vorticity decomposition. Color contour in the POD mode 3 resembles the 
elongated streamwise structures revealed in the POD mode 2 but more spatially fragmented. This indicated that these two modes probably formed a pair of modes with statistically similar spatial structures. The POD vorticity mode 4 revealed dominant flow structures with a shorter length scale and less orientated as compared to those in the POD modes 2 and 3. By using the DNS results, Ranjan et al. (2010, 2017) intensively studied the turbulent swept flow over a cylindrical wire placed on a wall that is a model of flow through the wirewrapped fuel pins. These authors showed the elongated coherent structures in the near-wall region, and concluded that these structures were similar to those observed in the wall region of turbulent boundary layers (Rajagopalan and Antonia, 1993). The DNS results of Ranjan et al. (2010) also showed that the elongated vortices overlapped since they traveled in the streamwise direction, yielding combined large scale structures with positive and negative tilt, and inclined angles with respect to the axial and wall-normal ( $z$-axis in this paper) directions. In the present study, due to the tilting and inclining, the large-scale eddies impinged on the surfaces of pins, wires and enclosure wall, and then broke down into small size structures. In figure [2, footprints of the coherent structures discussed in Ranjan et al. (2010, 2010) were revealed in the near-wall region, and these structures had the width comparable to the eddy size $D_{e}$. The large-scale structures appeared to align with the mean flow direction, and to coexist, interact and combine when they were generated from the elongated structures. The breakdown mechanism of the vortices and their interactions with the surfaces of pins and wires play an important role in the flow dynamics and heat transfer (Ranjan et al., 2010). Although the PIV measurements were done on the axial plane, the evolution and existence of the coherent structures can be confirmed in figure ए2. The results from the POD vorticity decomposition showed that the dominant flow structures were elongated and aligned in the streamwise direction. The low-order POD vorticity modes showed the smaller scale vortices that were originated from the breakdown of the large, elongated streamwise vortices.

\section{Conclusions}

In this paper, we presented the PIV measurements in the near-wall region in the 61-pin wire-wrapped hexagonal fuel bundle at the Reynolds number $R e=19,000$. Flow statistics computed from the PIV velocity fields, such as profiles of mean velocity, r.m.s fluctuating velocity, and contour maps of the flowfields, were discussed. The spatial-temporal velocity cross-correlations were analyzed; the integral length scales and convection velocity of the flow were estimated. The velocity correlations showed the highly correlated regions at large separation distances that indicated the streamwise convection of travelling vortices. In addition, we analyzed the spatial-temporal cross-correlations between the wall fluctuating 
pressure and fluctuating velocity fields obtained in the near-wall region. The contour maps of the cross-correlations showed that the wall fluctuating pressure and flow structures were strongly correlated, and the footprints of the coherent structures were captured when these large-scale structures evolved in the near-wall region. Finally, we applied the POD analysis to the vorticity snapshots obtained in the near-wall region to reveal the dominant flow structures. The POD decomposition revealed that the large-scale structures were elongated and aligned with the mean flow. Moreover, these coherent structures interacted, and impinged on the surfaces, and then broke into smaller ones.

\section{Acknowledgments}

This material is based upon work supported by the Department of Energy [National Nuclear Security Administration] under Award Number DE-NE0008321. This article was prepared as an account of work sponsored by an agency of the United States Government. Neither the United States Government nor any agency thereof, nor any of their employees, makes any warranty, express or implied, or assumes any legal liability or responsibility for the accuracy, completeness, or usefulness of any information, apparatus, product, or process disclosed, or represents that its use would not infringe privately owned rights. Reference herein to any specific commercial product, process, or service by trade name, trademark, manufacturer, or otherwise does not necessarily constitute or imply its endorsement, recommendation, or favoring by the United States Government or any agency thereof. The views and opinions of authors expressed herein do not necessarily state or reflect those of the United States Government or any agency thereof.

\section{References}

\section{References}

Ahmad, I. and Kim, K.-Y. (2006). Flow and convective heat transfer analysis using rans for a wire-wrapped fuel assembly, Journal of Mechanical Science and Technology 20(9): 1514-1524.

Berkooz, G., Holmes, P. and Lumley, J. L. (1993). The proper orthogonal decomposition in the analysis of turbulent flows, Annual Review of Fluid Mechanics 25(1): 539-575.

Boomsma, A., Bhattacharya, S., Troolin, D., Pothos, S. and Vlachos, P. (2016). A comparative experimental evaluation of uncertainty estimation methods for two-component piv, Measurement Science and Technology 27(9): 094006.

Charonko, J. J. and Vlachos, P. P. (2013). Estimation of uncertainty bounds for individual particle image velocimetry measurements from cross-correlation peak ratio, Measurement Science and Technology 24(6): 065301 . 
Cheng, S.-K. and Todreas, N. E. (1986). Hydrodynamic models and correlations for bare and wire-wrapped hexagonal rod bundlesbundle friction factors, subchannel friction factors and mixing parameters, Nuclear Engineering and Design 92(2): 227-251.

Cherry, N., Hillier, R. and Latour, M. (1984). Unsteady measurements in a separated and reattaching flow., Journal of Fluid Mechanics 144(1): 13.

Dumas, R. (1990). Observations on the boundary layer based on measured correlations with various improvements, Near-Wall Turbulence 1: 437-452.

Durgesh, V. and Naughton, J. (2010). Multi-time-delay lse-pod complementary approach applied to unsteady high-reynolds-number near wake flow, Experiments in Fluids 49(3): 571-583.

Eckstein, A. and Vlachos, P. P. (2009a). Assessment of advanced windowing techniques for digital particle image velocimetry (dpiv), Measurement Science and Technology 20(7): 075402.

Eckstein, A. and Vlachos, P. P. (2009b). Digital particle image velocimetry (dpiv) robust phase correlation, Measurement Science and Technology 20(5): 055401.

Falchi, M. and Romano, G. P. (2009). Evaluation of the performance of high-speed piv compared to standard piv in a turbulent jet, Experiments in Fluids 47(3): 509-526.

Fischer, P., Lottes, J., Siegel, A. and Palmiotti, G. (2007). Large eddy simulation of wire-wrapped fuel pins i: hydrodynamics of a single pin, Proceedings of $M$ and $C+S N A$.

Gajapathy, R., Velusamy, K., Selvaraj, P. and Chellapandi, P. (2015). Cfd investigation of effect of helical wire-wrap parameters on the thermal hydraulic performance of 217 fuel pin bundle, Annals of Nuclear Energy 77: 498-513.

Gurka, R., Liberzon, A. and Hetsroni, G. (2006). Pod of vorticity fields: A method for spatial characterization of coherent structures, International Journal of Heat and Fluid Flow 27(3): 416-423.

Holmes, P., Lumley, J. L. and Berkooz, G. (1998). Turbulence, coherent structures, dynamical systems and symmetry, Cambridge University Press.

Hudy, L. M., Naguib, A. and Humphreys, W. M. (2007). Stochastic estimation of a separated-flow field using wall-pressure-array measurements, Physics of Fluids 19(2): 024103.

Hussain, A. F. (1986). Coherent structures and turbulence, Journal of Fluid Mechanics 173: 303-356.

Jeong, J.-H., Yoo, J., Lee, K.-L. and Ha, K.-S. (2015). Three-dimensional flow phenomena in a wire-wrapped 37-pin fuel bundle for sfr, Nuclear Engineering and Technology 47(5): 523-533.

Jones, B., Planchon, H. and Hammersley, R. (1973). Turbulent correlation measurements in a two-stream mixing layer., AIAA Journal 11(8): 1146-1150.

Kerhervé, F. and Fitzpatrick, J. (2011). Measurement and analysis of the turbulent length scales in jet flows, Experiments in Fluids 50(3): 637-651.

Kiya, M. and Sasaki, K. (1983). Structure of a turbulent separation bubble, Journal of Fluid Mechanics 137: $83-113$.

Kostas, J., Soria, J. and Chong, M. (2005). A comparison between snapshot pod analysis of piv velocity and vorticity data, Experiments in Fluids 38(2): 146-160.

Lee, I. and Sung, H. J. (2002). Multiple-arrayed pressure measurement for investigation of the unsteady flow structure of a reattaching shear layer, Journal of Fluid Mechanics 463: 377-402.

Liberzon, A., Gurka, R., Tiselj, I. and Hetsroni, G. (2005). Spatial characterization of the numerically simulated vorticity fields of a flow in a flume, Theoretical and Computational Fluid Dynamics 19(2): 115125.

Merzari, E., Fischer, P., Yuan, H., Van Tichelen, K., Keijers, S., De Ridder, J., Degroote, J., Vierendeels, 
J., Doolaard, H., Gopala, V. et al. (2016). Benchmark exercise for fluid flow simulations in a liquid metal fast reactor fuel assembly, Nuclear Engineering and Design 298: 218-228.

Nguyen, T. D., Wells, J. C., Mokhasi, P. and Rempfer, D. (2010). Proper orthogonal decomposition-based estimations of the flow field from particle image velocimetry wall-gradient measurements in the backwardfacing step flow, Measurement Science and Technology 21(11): 115406.

Nguyen, T., Pellé, J., Harmand, S. and Poncet, S. (2012). Piv measurements of an air jet impinging on an open rotor-stator system, Experiments in Fluids 53(2): 401-412.

Novendstern, E. (1972). Turbulent flow pressure drop model for fuel rod assemblies utilizing a helical wire-wrap spacer system, Nuclear Engineering and Design 22(1): 28-42.

Perry, A. and Chong, M. (1993). Topology of flow patterns in vortex motions and turbulence, Eddy Structure Identification in Free Turbulent Shear Flows, Springer, pp. 339-361.

Pointer, W. D., Fischer, P., Siegel, A. and Smith, J. (2008). Rans-based cfd simulations of wire-wrapped fast reactor fuel assemblies, Proceedings of the 2008 International Congress on Advances in Nuclear Power Plants-ICAPP'08.

Raffel, M., Willert, C. E., Wereley, S. and Kompenhans, J. (2013). Particle image velocimetry: a practical guide, Springer.

Rainer, H., Sven, S., Nico, R., Christian, J. K., Andreas, S., Reinhard, G., Janos, A., Anni, R., Matteo, N., Michel, S., Christophe, C., Jean-Marc, F., Sricharan, S., Jean-Philippe, L., Chris, W., Joachim, K., Julio, S., Omid, A. and Callum, A. (2016). Coherent large scale structures in adverse pressure gradient turbulent boundary layers, 18th International Symposium on Application of Laser Techniques to Fluid Mechanics.

Rajagopalan, S. and Antonia, R. (1993). Structure of the velocity field associated with the spanwise vorticity in the wall region of a turbulent boundary layer, Physics of Fluids A: Fluid Dynamics (1989-1993) 5(10): 2502-2510.

Ranjan, R., Pantano, C. and Fischer, P. (2010). Direct simulation of turbulent swept flow over a wire in a channel, Journal of Fluid Mechanics 651: 165-209.

Ranjan, R., Pantano, C. and Fischer, P. (2011). Direct simulation of turbulent heat transfer in swept flow over a wire in a channel, International Journal of Heat and Mass Transfer 54(21): 4636-4654.

Rasu, N. G., Velusamy, K., Sundararajan, T. and Chellapandi, P. (2014). Simultaneous development of flow and temperature fields in wire-wrapped fuel pin bundles of sodium cooled fast reactor, Nuclear Engineering and Design 267: 44-60.

Rehme, K. (1973). Pressure drop correlations for fuel element spacers, Nuclear Technology 17(1): 15-23.

Rolfo, S., Péniguel, C., Guillaud, M. and Laurence, D. (2012). Thermal-hydraulic study of a wire spacer fuel assembly, Nuclear Engineering and Design 243: 251-262.

Romano, G. P. (1995). Analysis of two-point velocity measurements in near-wall flows, Experiments in Fluids 20(2): 68-83.

Ruiz, T., Sicot, C., Brizzi, L., Borée, J. and Gervais, Y. (2010). Pressure/velocity coupling induced by a near wall wake, Experiments in Fluids 49(1): 147-165.

Saxena, A., Bieder, U., Cadiou, T. and Viazzo, S. (2013). Large eddy simulation of turbulent flow in wire wrapped fuel pin bundles cooled by sodium, Advancements in Nuclear Instrumentation Measurement Methods and Their Applications (ANIMMA), 2013 3rd International Conference on, IEEE, pp. 1-8.

Sirovich, L. and Kirby, M. (1987). Low-dimensional procedure for the characterization of human faces, Journal of The Optical Society of America 4(3): 519-524. 
Spalart, P. R. (1988). Contributions of numerical simulation data bases to the physics, modeling, and measurement of turbulence, Advances In Turbulence p. 11.

Timmins, B. H., Wilson, B. W., Smith, B. L. and Vlachos, P. P. (2012). A method for automatic estimation of instantaneous local uncertainty in particle image velocimetry measurements, Experiments in Fluids 53(4): 1133-1147.

Ukeiley, L. and Murray, N. (2005). Velocity and surface pressure measurements in an open cavity, Experiments in Fluids 38(5): 656-671.

Welch, P. D. (1967). The use of fast fourier transform for the estimation of power spectra: A method based on time averaging over short, modified periodograms, IEEE Transactions on Audio and Electroacoustics 15(2): 70-73.

Westerweel, J. (1994). Efficient detection of spurious vectors in particle image velocimetry data, Experiments in Fluids 16(3-4): 236-247.

Wilson, B. M. and Smith, B. L. (2013). Uncertainty on piv mean and fluctuating velocity due to bias and random errors, Measurement Science and Technology 24(3): 035302. 